\title{
Electromagnetic properties of ${ }^{100} \mathrm{Mo}$ : Experimental results and theoretical description of quadrupole degrees of freedom
}

\author{
K. Wrzosek-Lipska, ${ }^{1,2}$ L. Próchniak, ${ }^{1}$ M. Zielińska, ${ }^{1,3}$ J. Srebrny, ${ }^{1}$ K. Hadyńska-Klęk,,${ }^{1,4}$ J. Iwanicki, ${ }^{1}$ M. Kisieliński, ${ }^{1,5}$ \\ M. Kowalczyk, ${ }^{1,4}$ P. J. Napiorkowski, ${ }^{1}$ D. Piętak, ${ }^{1,6}$ and T. Czosnyka ${ }^{1, *}$ \\ ${ }^{1}$ Heavy Ion Laboratory, University of Warsaw, PL-02-093 Warsaw, Poland \\ ${ }^{2}$ Instituut voor Kern- en Stralingsfysica, K.U. Leuven, Celestijnenlaan 200D, B-3001 Leuven, Belgium \\ ${ }^{3}$ IRFU/SPhN, CEA Saclay, F-91191 Gif-sur-Yvette, France \\ ${ }^{4}$ Institute of Experimental Physics, University of Warsaw, PL-00-681 Warsaw, Poland \\ ${ }^{5}$ National Center for Nuclear Research, PL-05-400 Swierk, Poland \\ ${ }^{6}$ Institute of Radioelectronics, Warsaw University of Technology, PL-00-665 Warsaw, Poland
}

(Received 26 July 2012; published 7 December 2012)

\begin{abstract}
The Coulomb excitation experiment to study electromagnetic properties of the heaviest stable Mo isotope, ${ }^{100} \mathrm{Mo}$, was performed using a $76 \mathrm{MeV}{ }^{32} \mathrm{~S}$ beam from the Warsaw cyclotron U-200P. Magnitudes and relative signs of $26 E 1, E 2, E 3$, and $M 1$ matrix elements coupling nine low-lying states in ${ }^{100}$ Mo were determined using the least-squares code GOSIA. Diagonal matrix elements (related to the spectroscopic quadrupole moments) of the $2_{1}^{+}, 2_{2}^{+}$, and $2_{3}^{+}$states as well as the $4_{1}^{+}$state were extracted. The resulting set of reduced $E 2$ matrix elements was complete and precise enough to obtain, using the quadrupole sum rules approach, quadrupole deformation parameters of ${ }^{100} \mathrm{Mo}$ in its two lowest $0^{+}$states: ground and excited. The overall deformation of the $0_{1}^{+}$and $0_{2}^{+}$ states in ${ }^{100} \mathrm{Mo}$ is of similar magnitude, in both cases larger compared to what was found for the neighboring isotopes ${ }^{96} \mathrm{Mo}$ and ${ }^{98} \mathrm{Mo}$. At the same time, the asymetry parameters obtained for both states strongly differ, indicating a triaxial shape of the ${ }^{100} \mathrm{Mo}$ nucleus in the ground state and a prolate shape in the excited $0^{+}$state. Low-energy quadrupole excitations of the ${ }^{100}$ Mo nucleus were studied in the frame of the general quadrupole collective Bohr Hamiltonian model (GBH). The potential energy and inertial functions were calculated using the adiabatic time-dependent Hartree-Fock-Bogoliubov (ATDHFB) method starting from two possible variants of the Skyrme effective interaction: SIII and Sly4. The overall quadrupole deformation parameters resulting from the GBH calculations with the SLy4 variant of the Skyrme interaction are slightly closer to the experimentally obtained values than those obtained using SIII.
\end{abstract}

DOI: 10.1103/PhysRevC.86.064305

PACS number(s): 25.70.De, 21.60.Ev, 23.20.-g, 27.60.+j

\section{INTRODUCTION}

In the transitional region of the nuclear chart $(A \sim 100)$ shape instabilities, which may lead to coexisting nuclear shapes, are relatively common. For $\mathrm{Sr}(Z=38)$ and $\mathrm{Zr}$ $(Z=40)$ isotopic chains a dramatic drop of the energy of the $2_{1}^{+}$state was observed around neutron number $N=60$ (Figs. 1 and 2), indicating a sudden shape transition. Moreover, E0 transition strengths measured for these isotopes suggest that the ground-state structure changes rapidly when the neutron number increases from $N=58$ to $N=60$ [1]. In the Mo isotopes $(Z=42)$ the change of the energy of the $2_{1}^{+}$state is less pronounced, but large magnitudes of experimentally determined $E 0$ transition strengths may indicate the presence and mixing of different nuclear shapes. According to Rodriguez-Guzman et al. [2], the smooth evolution of nuclear properties, observed for the deformation, charge radii, and nuclear binding energies of Mo isotopes, may have its origin in an emergent region of triaxiality.

The structure of Mo isotopes with neutron number greater than 50 provides a challenge to theoretical models. Level schemes of even-even $50 \leqslant N \leqslant 64$ molybdenum isotopes (limited to low-lying excited states) are presented in Fig. 1.

${ }^{*}$ Deceased
The lightest stable isotope ${ }^{92} \mathrm{Mo}_{50}$ is spherical [3], has a closed $N=50$ neutron shell, and its low-energy excited states arise from neutrons and protons interacting in $g_{7 / 2}$ and $g_{9 / 2}$ orbits. The $2_{1}^{+}$level energy systematics as well as evolution of the reduced transition probabilities $B\left(E 2 ; 2_{1}^{+} \rightarrow 0_{1}^{+}\right)$for the even-even Mo isotopes (Fig. 3) suggest that with increasing neutron number the influence of the collective motion on the electromagnetic structure becomes stronger. This effect is visible between $N=56$ and $N=60$. In consequence, the first unstable Mo isotope, ${ }^{102} \mathrm{Mo}_{60}$, has a vibrational-like structure. The structure of heavy stable molybdenum isotopes, ${ }^{96} \mathrm{Mo}_{54},{ }^{98} \mathrm{Mo}_{56}$, and ${ }^{100} \mathrm{Mo}_{58}$, arises from competition between single-particle and collective motion, resulting in unusual features of these nuclei.

Among stable even-even nuclei one can find only few where the first excited state has a spin and parity $0^{+}$. In only four cases the energy of the $0^{+}$state does not exceed $2 \mathrm{MeV}$. One of these unusual nuclei is the ${ }^{98}$ Mo isotope and two others, ${ }^{90,96} \mathrm{Zr}$, also lie close to the region of rapid shape changes. In the ${ }^{100} \mathrm{Mo}$ isotope the $0_{2}^{+}$state is observed at an energy of $695 \mathrm{keV}$, very close to the first $2^{+}$state $(536 \mathrm{keV})$. Description of such a rare structure in the framework of existing nuclear structure models is still difficult. On the other hand, the observation of low-lying $0^{+}$excited states can be the first indication of the shape coexistence phenomenon. This effect was recently confirmed in the neighboring ${ }^{98} \mathrm{Mo}$ isotope where 


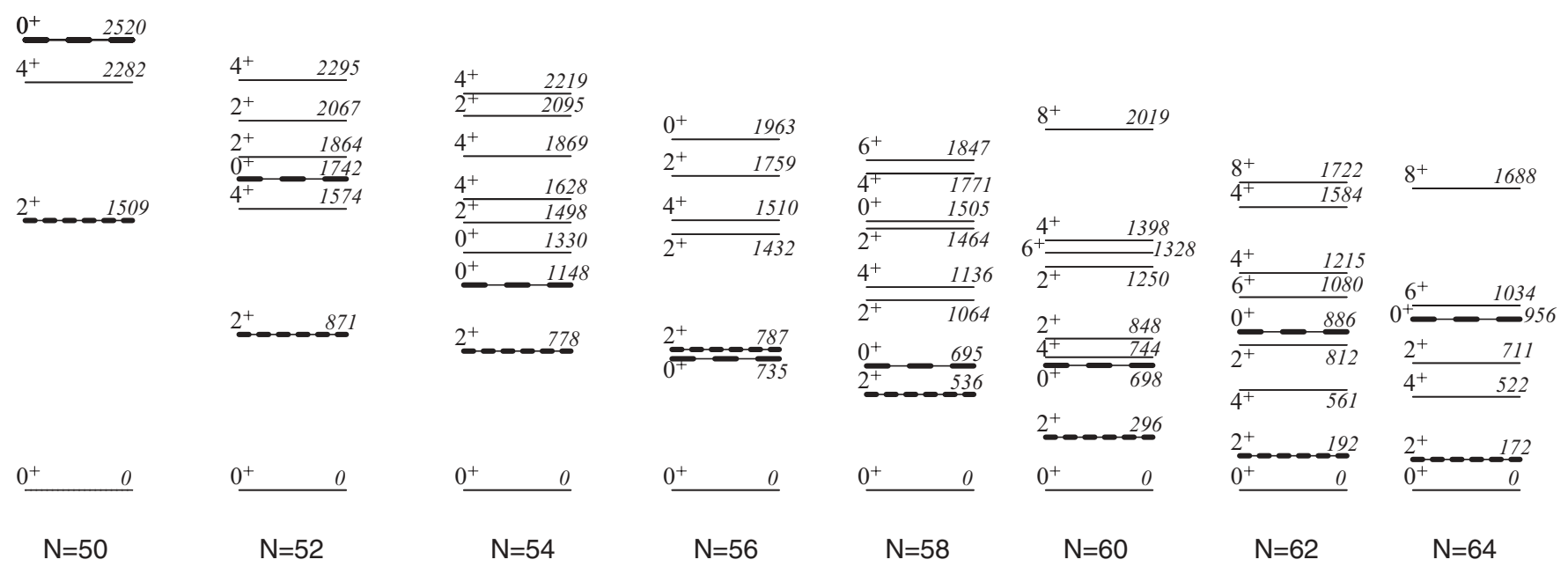

FIG. 1. Low-energy parts of level schemes for even-even molybdenum isotopes $(50 \leqslant N \leqslant 64)$. Level energies are given in keV. The first excited $2^{+}$and $0^{+}$states are marked with dashed lines. The $0_{2}^{+}$state in ${ }^{100}$ Mo lies very close in energy to the $2_{1}^{+}$state, while it becomes even the first excited one in the neighboring ${ }^{98}$ Mo isotope.

shape coexistence manifests in a very different triaxiality of the two first $0^{+}$states [4].

Multiple Coulomb excitation is one of the most important experimental methods to study nuclear collectivity and shapes. While lifetime measurements allow determination of reduced transition probabilities, the Coulomb excitation technique can provide information on relative signs of the matrix elements. Moreover, it is sensitive to diagonal matrix elements via second-order effects, making it possible to extract quadrupole moments including their signs, which are related to the shape of the nucleus in a given state. Low-energy Coulomb excitation is the only experimental technique that can distinguish between prolate and oblate shapes of the nucleus in its intrinsic frame without any assumptions based on nuclear structure models.

This paper presents results of the Coulomb excitation study of the ${ }^{100} \mathrm{Mo}$ isotope, in particular those concerning

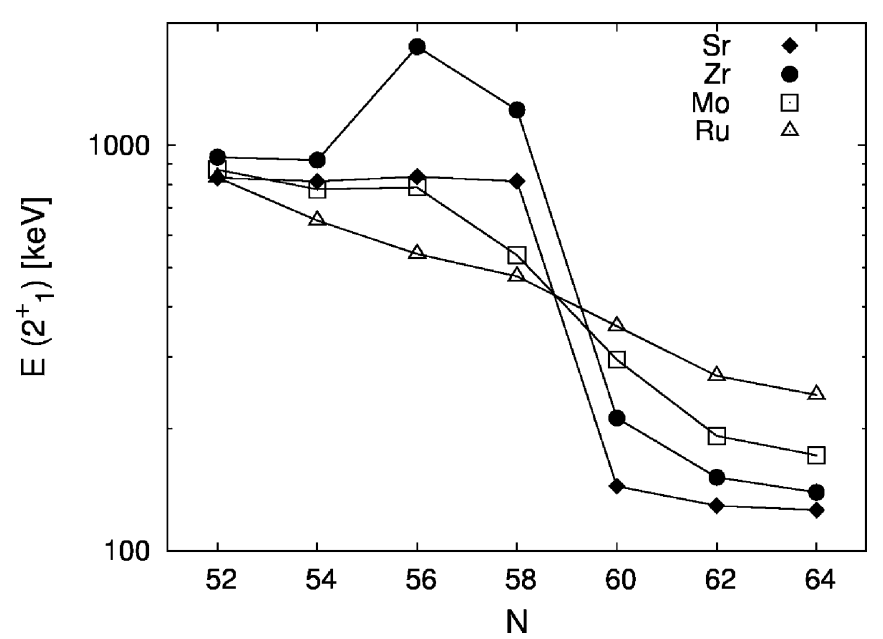

FIG. 2. Excitation energy of the $2_{1}^{+}$state as a function of neutron number in $\mathrm{Zr}, \mathrm{Sr}, \mathrm{Mo}$, and Ru isotopes. A dramatic drop of the energy of the $2_{1}^{+}$state in $\mathrm{Zr}$ and $\mathrm{Sr}$ isotopes is visible between $N=56$ and $N=60$. quadrupole deformation parameters of the two lowest $0^{+}$states in this nucleus. It is organized as follows: results of earlier experiments to study electromagnetic properties of ${ }^{100} \mathrm{Mo}$ are presented in Sec. II, experimental details in Sec. III, selected aspects of the Coulomb excitation data analysis are reported in Sec. IV, and results are presented in Sec. V. In Sec. VI the quadrupole sum rules method is described, and experimentally derived quadrupole shape parameters of the low-lying $0^{+}$states in ${ }^{100} \mathrm{Mo}$ are presented.

\section{PREVIOUS RESEARCH ON ${ }^{100} \mathrm{MO}$}

The level scheme of ${ }^{100} \mathrm{Mo}$ is well known from numerous studies of the $\gamma$ radiation following the $\beta$ decay of ${ }^{100} \mathrm{Nb}$ and reactions produced by light particles $(n, p, d, \alpha)$. The

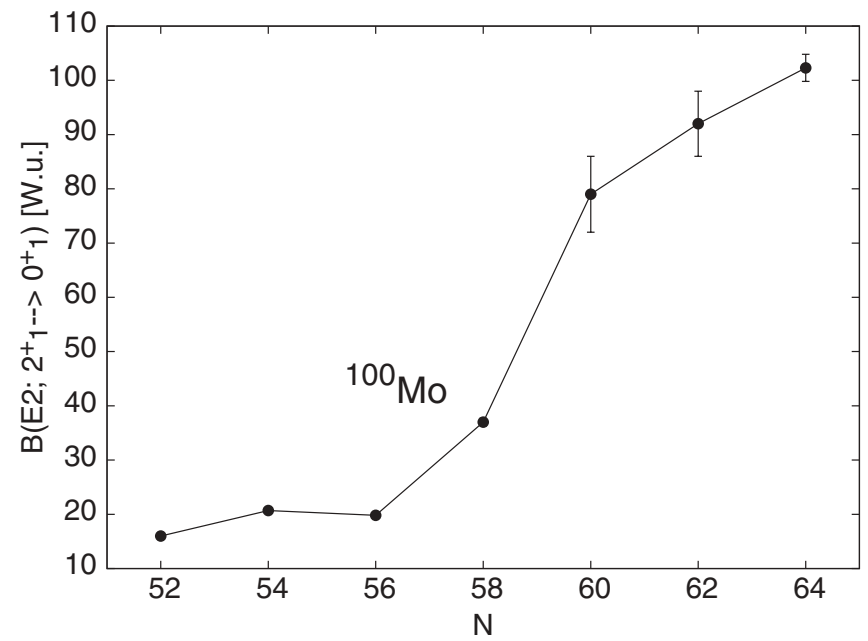

FIG. 3. Reduced transition probability $B\left(E 2 ; 2_{1}^{+} \rightarrow 0_{1}^{+}\right)$as a function of neutron number in even-even molybdenum isotopes. A sudden increase of the $B\left(E 2 ; 2_{1}^{+} \rightarrow 0_{1}^{+}\right)$value is observed between $N=56$ and $N=60$. 
information on high spin states in ${ }^{100}$ Mo was obtained in heavy-ion reactions induced by ${ }^{136} \mathrm{Xe} \mathrm{[5],}{ }^{86} \mathrm{Kr}$ [6], and ${ }^{30} \mathrm{Si} \mathrm{[7].}$

From this type of measurement branching ratios and $E 2 / M 1$ mixing ratios were determined, including those of transitions between the low-lying states of ${ }^{100} \mathrm{Mo}$, which were populated in the experiment described in Sec. III of the present paper.

Some reduced transition probabilities in the ${ }^{100}$ Mo nucleus were known from previous Coulomb excitation measurements [8,9]. In the experiment of Barrette et al. [8] a thick ${ }^{100} \mathrm{Mo}$ target was bombarded with light beams of ${ }^{16} \mathrm{O}$ ions and alpha particles. The $\gamma$ rays, depopulating Coulomb excited states of ${ }^{100} \mathrm{Mo}$, were detected without coincidences with scattered particles. From the measured $\gamma$-ray yields Coulomb excitation cross sections and transition probabilities were calculated. For this purpose very precise information on the absolute detection efficiency, beam current, electronics dead time, stopping powers, and other factors influencing measured $\gamma$-ray intensities were needed. The influence of quadrupole moments of the excited states in ${ }^{100}$ Mo on their population was not taken into account. Moreover, the energy of the incident ${ }^{16} \mathrm{O}$ beam, equal to $44.8 \mathrm{MeV}$, exceeded the safe beam energy (40 MeV for the backscattering of ${ }^{16} \mathrm{O}$ on ${ }^{100} \mathrm{Mo}$ ) and in consequence the excitation of the ${ }^{100}$ Mo nuclei could not be considered as a purely electromagnetic process. This conclusion is supported by the results of Bohn et al. [11], who observed that for the ${ }^{16} \mathrm{O}+{ }^{100} \mathrm{Mo}$ system the elastic cross section becomes smaller than the Rutherford cross section above $40 \mathrm{MeV}$, indicating the onset of nuclear absorption. This may explain the discrepancy between the value of the $\left\langle 2_{1}^{+}\|E 2\| 0_{1}^{+}\right\rangle$matrix element equal to $0.725(18) e b$, reported by Ref. [8], and results of other experiments, such as lifetime measurement [11] [0.68(1) eb] or the present work [0.689(17) $\mathrm{eb}$.

A set of $18 E 2$ reduced matrix elements coupling low-lying states of ${ }^{100}$ Mo was determined by Mundy et al. [9] in a combination of two complementary experiments: Coulomb excitation and lifetime measurement. For the first one, a ${ }^{100} \mathrm{Mo}$ target was Coulomb excited using beams of ${ }^{16} \mathrm{O}$ and ${ }^{32} \mathrm{~S}$ at 42 and $89 \mathrm{MeV}$, respectively. Emitted $\gamma$ rays were observed in coincidence with Coulomb scatterred beam particles. The second measurement applied the recoil-distance method to study lifetimes of excited states populated by the Coulomb excitation of ${ }^{100} \mathrm{Mo}$ by a ${ }^{58} \mathrm{Ni}$ beam of $185 \mathrm{MeV}$ energy. The final set of matrix elements, obtained in Ref. [9], was deduced both from the particle- $\gamma$ coincidence yields as well as from the measured lifetimes. The data were analyzed using the semiclassical Coulomb excitation code of Winther and de Boer [12], which calculates excitation amplitudes and Coulomb cross sections for a given set of electromagnetic multipole matrix elements. The inversion of the problem, i.e., the extraction of the matrix elements from the observed $\gamma$-ray yields, is not straightforward due to complicated nonlinear dependencies. In contrast to analysis of the Coulomb excitation data presented in the present paper, no minimization process was applied. The signs of all transitional E2 matrix elements were taken as predicted by the IBM-2 model [10]. As it is not clear how these signs were determined, in particular why the signs of in-band transitional matrix elements change within the band, only absolute values of transitional matrix elements were used for comparison with the results of the present work, discussed in Sec. V.

The spectroscopic quadrupole moment of the first $2^{+}$ state of ${ }^{100}$ Mo was obtained using the reorientation effect in Coulomb excitation $[13,14]$. The data were analyzed using the code of Winther and de Boer [12], assuming values of transitional E2 matrix elements from Ref. [8].

All lifetimes of low-lying excited states in ${ }^{100} \mathrm{Mo}$ are in the picosecond range, except for the $0_{2}^{+}$state that has a lifetime of several nanoseconds. The picosecond lifetmes of the $2_{1}^{+}, 2_{2}^{+}$, $2_{3}^{+}, 4_{1}^{+}, 4_{2}^{+}, 6_{1}^{+}$, and $3^{-}$states in ${ }^{100}$ Mo were measured using the recoil-distance method [9,11], while the lifetime of the isomeric $0_{2}^{+}$state was determined via the $\beta-\gamma-\gamma$ fast-timing technique [15-17].

Up to now there was no experimental information concerning the quadrupole deformation parameters of ${ }^{100} \mathrm{Mo}$, in particular the triaxiality of this nucleus in its ground and the first excited $0^{+}$state. Such information is necessary to conclude on the occurrence of the shape coexistence phenomenon in ${ }^{100} \mathrm{Mo}$, which was predicted in this mass region by Wood et al. [1] and observed experimentally in the neighboring ${ }^{98} \mathrm{Mo}$ [4].

\section{EXPERIMENTAL DETAILS}

The Coulomb excitation experiment to study ${ }^{100}$ Mo was performed at the Heavy Ion Laboratory, University of Warsaw, using a dedicated Coulomb excitation setup [18].

A ${ }^{32} \mathrm{~S}$ beam of $76 \mathrm{MeV}$ energy, delivered by the U-200P cyclotron, was impinging on a ${ }^{100}$ Mo target $44 \mathrm{mg} / \mathrm{cm}^{2}$ thick. The beam energy was chosen below the "safe" value [19], i.e., it fulfilled the condition of the closest approach to be greater than

$$
E_{\mathrm{safe}}=1.25\left(A_{p}^{1 / 3}+A_{t}^{1 / 3}\right)+5 \mathrm{fm} .
$$

This requirement ensures a purely electromagnetic interaction.

The recoiling target nuclei were stopped in the target material and Doppler shift correction of $\gamma$-ray energies was not necessary.

The $\gamma$ rays depopulating Coulomb-excited states were detected by the OSIRIS-II array. The OSIRIS-II spectrometer was a multidetector system consisting of 12 high-purity germanium (HPGe) detectors equipped with anti-Compton BGO shields. Germanium detectors were placed at a distance of $16 \mathrm{~cm}$ from the target, at the following laboratory angles with respect to the beam direction: $25^{\circ}$ ( $1 \mathrm{Ge}$ detector), $38^{\circ}$ $(2), 63^{\circ}(2), 90^{\circ}(2), 117^{\circ}(2), 142^{\circ}(2)$, and $155^{\circ}(1)$.

In order to exploit the strong dependence of Coulomb excitation cross section on the particle scattering angle, data were collected for several angular ranges. In this way independent data sets sensitive to all relevant matrix elements were obtained. The scattered projectiles were detected by 45 small silicon detectors, $\mathrm{PiN}$ diodes, placed inside a compact spherical chamber of $5 \mathrm{~cm}$ radius [20] used previously with the NORDBALL setup [21]. A relatively small active area of a single PiN-diode detector $\left(0.5 \times 0.5 \mathrm{~cm}^{2}\right)$ provides information on particle scattering angle that is sufficiently 

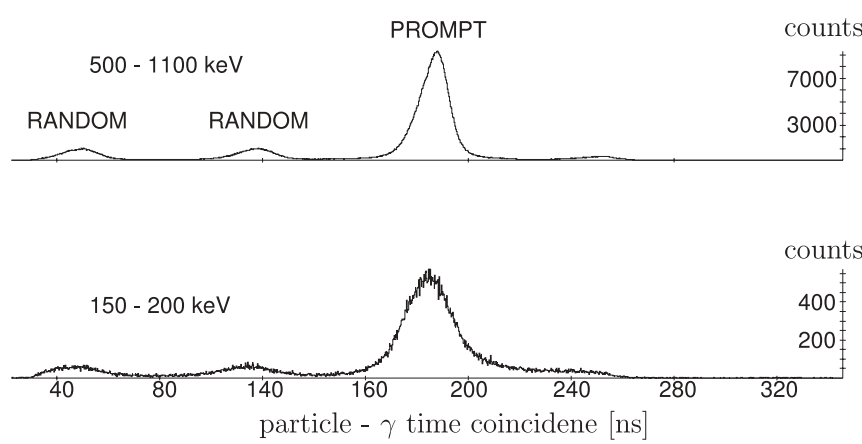

FIG. 4. Particle- $\gamma$ coincidence time spectrum presented for different gamma energy gates: $150-200 \mathrm{keV}$ and 500-1100 keV. Prompt and random coincidence peaks are marked.

precise to properly describe the excitation process. Scattering laboratory angle coverage extended from $110^{\circ}$ to $152^{\circ}$ with respect to the beam direction.

The data were collected with a coincidence requirement between $\gamma$ rays detected in OSIRIS II and backscattered ${ }^{32} \mathrm{~S}$ ions. Events were collected under the condition that at least one $\gamma$ ray was detected in the OSIRIS II spectrometer in coincidence with exactly one scattered ${ }^{32} \mathrm{~S}$ ion detected by a PiN diode.

Time-coincident particle- $\gamma$ data were collected within a 400 ns coincidence window. Particle-gamma coincidence spectra are presented in Fig. 4 separately for high-energy (500 $1100 \mathrm{keV})$ and low-energy (150-200 keV) $\gamma$ rays. Decrease of time resolution with energy due to charge collection time is clearly visible. The distance of $70 \mathrm{~ns}$ between the maxima present in the time spectra is related to the pulsed structure of the beam delivered by the Warsaw cyclotron. Prompt coincidences were selected in the offline analysis using a $50 \mathrm{~ns}$ gate in the time spectrum. Random coincidences were subtracted, with special attention paid to low-energy transitions, as the proper estimation of the random events related to the $0_{2}^{+} \rightarrow 2_{1}^{+} \gamma$ ray transiton of $159 \mathrm{keV}$ energy was crucial to determine the $\left\langle 2_{1}^{+}\|E 2\| 0_{2}^{+}\right\rangle$matrix element, important in the further analysis aiming at the extraction of the quadrupole deformation parameters in ${ }^{100} \mathrm{Mo}$.

\section{COULOMB EXCITATION ANALYSIS}

Choosing the right strategy to subdivide the Coulomb excitation data is an important issue during the data analysis [22]. In a multiple Coulomb excitation process several levels are observed and number of unknown matrix elements affects Coulomb excitation cross sections in a complicated nonlinear way. Subdivision of the experimental data based on the projectile scattering angle makes it possible to disentangle contributions from various excitation paths.

To demonstrate the dependence of the relative population of a state on the scattering angle, the example of the $0_{2}^{+}$state in ${ }^{100} \mathrm{Mo}$ is shown in Fig. 5. The $0_{2}^{+}$state at the energy of $695 \mathrm{keV}$ can be populated only via two-step Coulomb excitation: $\left(0_{1}^{+} \rightarrow 2_{1}^{+}\right) \otimes\left(2_{1}^{+} \rightarrow 0_{2}^{+}\right)$. The probability of the two-step process with respect to the one-step excitation of the $2_{1}^{+}$state in ${ }^{100} \mathrm{Mo}$ increases with the projectile scattering angle. In addition, it is clearly visible that the population of

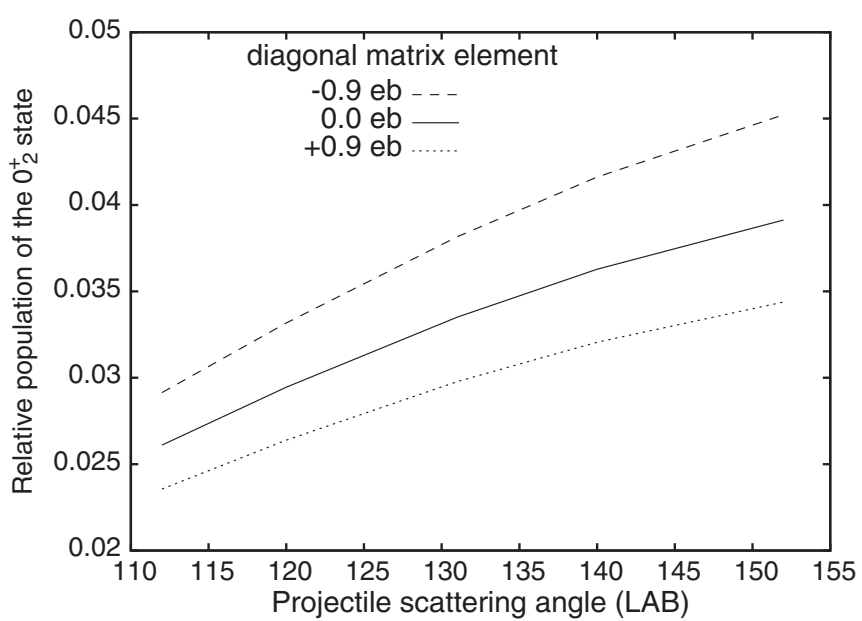

FIG. 5. Relative population of the $0_{2}^{+}$state (normalized to the population of the $2_{1}^{+}$state) as a function of the projectile scattering angle, calculated for three values of the $2_{1}^{+}$diagonal matrix element: $-0.9 \mathrm{eb}$ (dashed curve), $0.9 \mathrm{eb}$ (dotted curve), and $0.0 \mathrm{eb}$ (solid curve); the $0.9 \mathrm{eb}$ value corresponds to the axially symmetric rigid rotor limit. Relative population of the $0_{2}^{+}$state increases with the projectile scattering angle and is largest for the negative value of the diagonal matrix element of the $2_{1}^{+}$state.

the $\mathrm{O}_{2}^{+}$state is also sensitive to the quadrupole moment of the intermediate $2_{1}^{+}$state.

When analyzing the data from the Coulomb excitation experiment of ${ }^{100} \mathrm{Mo}$ with the ${ }^{32} \mathrm{~S}$ beam, it was decided to sum the spectra from individual Ge detectors. In this way the number of counts in observed $\gamma$ lines was increased to a

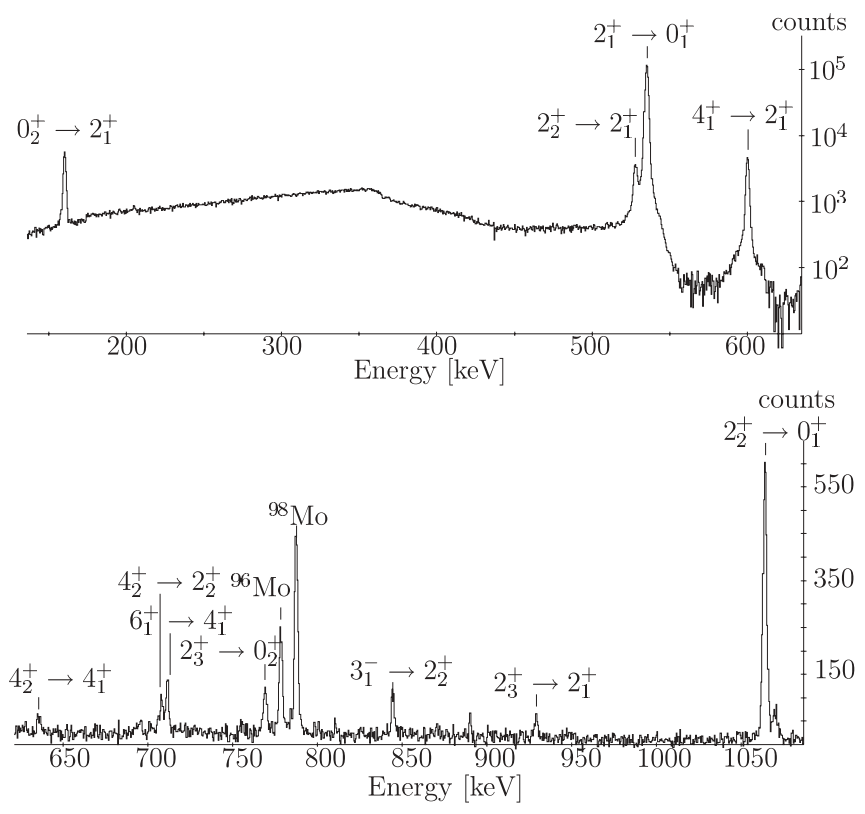

FIG. 6. A spectrum of $\gamma$ rays following the Coulomb excitation of ${ }^{100} \mathrm{Mo}$ with the ${ }^{32} \mathrm{~S}$ beam, collected in coincidence with beam particles scattered in the range between 112 and 124 degrees. The low-energy part of the spectrum is shown on the upper figure, and the high-energy part of the spectrum is shown on the lower figure. Random coincidences were subtracted. 


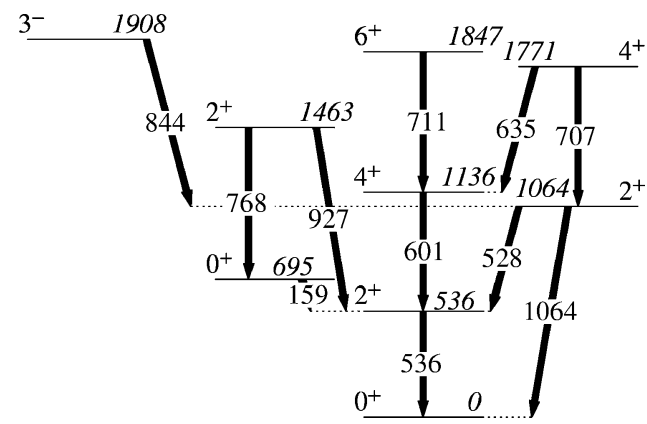

FIG. 7. Low-energy part of the level scheme for the ${ }^{100}$ Mo isotope showing $\gamma$-ray transitions observed in the present Coulomb excitation experiment. All energies of $\gamma$-ray transitions and levels are given in $\mathrm{keV}$.

level sufficient to subdivide the data according to the projectile scattering angle. Three ranges of the ${ }^{32} \mathrm{~S}$ scattering angle were used: $112^{\circ}-124^{\circ}, 127^{\circ}-131^{\circ}$, and $135^{\circ}-152^{\circ}$.

Figure 6 shows an example of a $\gamma$-ray spectrum of ${ }^{100} \mathrm{Mo}$ collected in coincidence with ${ }^{32} \mathrm{~S}$ ions scattered at $112^{\circ}-124^{\circ}$ laboratory angles. Figure 7 presents the low-energy part of the ${ }^{100}$ Mo level scheme that was populated in the experiment, together with the observed electromagnetic transitions.

\section{A. Extraction of reduced matrix elements}

To determine $E 1, E 2, E 3$, and $M 1$ reduced matrix elements in ${ }^{100} \mathrm{Mo}$, the Coulomb-excitation least-squares fitting code GOSIA [23] was used. The code fits a set of reduced matrix elements to the measured $\gamma$-ray yields taking into account known spectroscopic data related to electromagnetic matrix elements: branching ratios, lifetimes, $E 2 / M 1$ mixing ratios, as well as previously measured matrix elements. Those data are used as additional data points, entering the $\chi^{2}$ function on an equal basis as the gamma-ray yields observed in the Coulomb excitation experiment. Tables I, II, and III list the spectroscopic data that were included in the Coulomb excitation analysis. Branching ratio and mixing ratio $\delta(E 2 / M 1)$ values were taken from Refs. [7,8,24-27]. For lifetimes, only results of direct lifetime measurements were used $[9,11,15]$ and not the $B(E 2)$ 's resulting from Coulomb excitation experiments.

The GOSIA code constructs a standard $\chi^{2}$ function built of $\gamma$ ray yields measured in the Coulomb excitation experiment and

TABLE I. Half lives of the excited states populated in the Coulomb excitation experiment, taken into account in the current analysis.

\begin{tabular}{lcc}
\hline \hline State & $T_{1 / 2}(\mathrm{ps})$ & Reference \\
\hline $2_{1}^{+}$ & $13.6 \pm 0.7$ & {$[11]$} \\
$0_{2}^{+}$ & $1580 \pm 40$ & {$[15]$} \\
$2_{2}^{+}$ & $6.45 \pm 0.58$ & {$[9]$} \\
$4_{1}^{+}$ & $3.83 \pm 0.34$ & {$[9]$} \\
$2_{3}^{+}$ & $2.93 \pm 0.68$ & {$[9]$} \\
$4_{2}^{+}$ & $2.45 \pm 0.41$ & {$[9]$} \\
$6_{1}^{+}$ & $1.20 \pm 0.17$ & {$[9]$} \\
$3_{1}^{-}$ & $12.0 \pm 3.0$ & {$[9]$} \\
\hline \hline
\end{tabular}

TABLE II. Mixing ratios $\delta(E 2 / M 1)$ [24-27] which were taken into account in the current analysis.

\begin{tabular}{lr}
\hline \hline Transition & $\delta(E 2 / M 1)$ \\
\hline $2_{2}^{+} \rightarrow 2_{1}^{+}$ & $4.4 \pm 1.5$ \\
$2_{3}^{+} \rightarrow 2_{1}^{+}$ & $-0.27 \pm 0.02$ \\
\hline \hline
\end{tabular}

those calculated from a given set of matrix elements, as well as experimental and calculated values of known spectroscopic data.

For a given scattering kinematics, defined by the detection array geometry, and a given set of matrix elements, Coulomb excitation amplitudes for the investigated nucleus as well as $\gamma$ ray decay of states populated in the process are calculated. The calculation includes the effects influencing $\gamma$-ray intensities, such as internal conversion, and the finite size and relative efficiency of Ge detectors. An exact reproduction of the experimentally observed $\gamma$-ray intensities requires integration over a finite scattering angle range and over the range of bombarding energies resulting from the projectile energy loss in a target.

As a result of minimization of the $\chi^{2}$ function, a set of 26 reduced matrix elements with relative signs has been found to optimally reproduce the experimental data. The relative signs of matrix elements have a significant influence on Coulomb excitation probabilities. A given excited state can be populated in a one-step process that is described by a single matrix element, or indirectly via other excited states, when two or more matrix elements are involved. For each excitation path the contribution to the total excitation amplitude is proportional to the product of the relevant matrix elements. The excitation probability is the square of the sum of excitation amplitudes, and therefore it contains not only quadratic terms, but also interference terms between possible excitation paths. Signs of the interference terms depend on the relative signs of matrix elements. In the present work the signs of wave functions of excited nuclear states have been chosen in such a way that all $E 2$ matrix elements for transitions in the ground-state band, as well as for the $4_{2}^{+} \rightarrow 2_{2}^{+}$and $2_{3}^{+} \rightarrow 0_{2}^{+}$transitions, are assumed to be positive. The positive sign has been chosen also for the interband matrix elements for the $0_{2}^{+} \rightarrow 2_{1}^{+}, 2_{2}^{+} \rightarrow 2_{1}^{+}$, and $3^{-} \rightarrow 2_{1}^{+}$transitions. The signs of remaining matrix elements were determined relative to those.

TABLE III. Branching ratios [7,8,24-26] which were taken into account in the current analysis.

\begin{tabular}{lcc}
\hline \hline Transition & Energy $(\mathrm{keV})$ & Branching ratio \\
\hline $2_{2}^{+} \rightarrow 0_{1}^{+} / 2_{2}^{+} \rightarrow 2_{1}^{+}$ & $1064 / 528$ & $0.38 \pm 0.01$ \\
$2_{2}^{+} \rightarrow 0_{2}^{+} / 2_{2}^{+} \rightarrow 2_{1}^{+}$ & $369 / 528$ & $0.017 \pm 0.002$ \\
$2_{3}^{+} \rightarrow 4_{1}^{+} / 2_{3}^{+} \rightarrow 0_{2}^{+}$ & $327 / 768$ & $0.035 \pm 0.015$ \\
$2_{3}^{+} \rightarrow 2_{2}^{+} / 2_{3}^{+} \rightarrow 0_{2}^{+}$ & $399 / 768$ & $0.058 \pm 0.011$ \\
$2_{3}^{+} \rightarrow 2_{1}^{+} / 2_{3}^{+} \rightarrow 0_{2}^{+}$ & $927 / 768$ & $0.73 \pm 0.01$ \\
$4_{2}^{+} \rightarrow 2_{2}^{+} / 4_{2}^{+} \rightarrow 4_{1}^{+}$ & $707 / 635$ & $0.55 \pm 0.03$ \\
$3_{1}^{-} \rightarrow 0_{1}^{+} / 3_{1}^{-} \rightarrow 2_{2}^{+}$ & $1908 / 844$ & $0.045 \pm 0.01$ \\
$3_{1}^{-} \rightarrow 2_{1}^{+} / 3_{1}^{-} \rightarrow 2_{2}^{+}$ & $1372 / 844$ & $0.46 \pm 0.04$ \\
\hline \hline
\end{tabular}


The relative signs of matrix elements have a significant influence on Coulomb excitation probabilities. The signs and magnitudes of matrix elements reported in the present work were precisely verified by performing the minimization process with various initial values and comparing the quality of the obtained solutions. This will be illustrated by the example of the $\left\langle 2_{2}^{+}\|E 2\| 2_{2}^{+}\right\rangle$diagonal matrix element:

(i) Imposing a negative sign on the $\left\langle 2_{2}^{+}\|E 2\| 2_{2}^{+}\right\rangle$matrix element results in an immediate fivefold rise of the $\chi^{2}$ value caused mostly by a disagreement of calculated/experimental $\gamma$-ray yields for transitions deexciting the $2_{2}^{+}$state. Subsequent minimization restores the positive sign of the diagonal matrix element.

(ii) An attempt to reduce the value of the $\left\langle 2_{2}^{+}\|E 2\| 2_{2}^{+}\right\rangle$ matrix element reduces the population of the $2_{2}^{+}$ state, thus reducing the calculated $\gamma$-ray yields for the $2_{2}^{+} \rightarrow 2_{1}^{+}$and $2_{2}^{+} \rightarrow 0_{1}^{+}$transitions deexciting this state. The effect may be compensated by increasing corresponding transitional matrix elements $\left\langle 2_{2}^{+}\|E 2\| 2_{1}^{+}\right\rangle$and $\left\langle 2_{2}^{+}\|E 2\| 0_{1}^{+}\right\rangle$, but this immediately causes disagreement with the experimental value of the $2_{2}^{+}$state lifetime [9].

Statistical errors of the matrix elements were estimated after the convergence of the $\chi^{2}$ function had been achieved. The applied method, suitable for error calculation in large, strongly coupled, nonlinear systems, involves two steps. First, the multidimensional $\chi^{2}$ surface is sampled for various values of the given matrix element in the vicinity of the minimum in order to estimate its "diagonal" statistical uncertainty. Then the total statistical error including the dependence on the other matrix elements (cross correlation effects) is calculated. The total error is obtained by requiring the total integrated probability distribution in the space of fitted parameters to be equal to the confidence limit, $68.3 \%$ [23].

\section{RESULTS}

Magnitudes of reduced matrix elements with their relative signs deduced from the current analysis are presented in Tables IV, V (transitional and diagonal E2 matrix elements), and VI (transitional $M 1$ matrix elements).

It should be noted that all matrix elements between the $0_{2}^{+}$state and $2_{1,2,3}^{+}$states, which were extracted in the present work, indicate stronger couplings than were pointed out in Ref. [9]. The present value of the $\left\langle 2_{1}^{+}\|E 2\| 0_{2}^{+}\right\rangle$matrix element is larger than reported in previous works $[8,9]$ where it was assumed that the deexcitation of the $0_{2}^{+}$state proceeds uniquely by the gamma decay to the $2_{1}^{+}$state. In reality, a significant $E 0$ branch was observed in electron spectroscopy measurements, with branching ratio $\left(E 0 ; 0_{1}^{+} \rightarrow 0_{2}^{+}\right) /\left(E 2 ; 0_{2}^{+} \rightarrow 2_{1}^{+}\right)$equal to $0.110(8)$ [17], 0.127(8) [29], and 0.086 [30]. The weighted average of these three values was included in the present analysis.

One may also notice that different values, compared to the previous results, were presently obtained for matrix elements that couple the $2_{3}^{+}$state with the $2_{1}^{+}$and $4_{1}^{+}$states. Mundy et al. [9] claim that the coupling between the $4_{1}^{+}$and $2_{3}^{+}$states
TABLE IV. Reduced nondiagonal E2 matrix elements in ${ }^{100} \mathrm{Mo}$ obtained in the present work, compared to the E2 matrix elements determined previously [calculated from $B(E 2)$ values from Refs. [8,9,11], assuming a positive sign].

\begin{tabular}{lcllc}
\hline \hline$I_{i} \rightarrow I_{f}$ & \multicolumn{4}{c}{$\left\langle I_{f}\|E 2\| I_{i}\right\rangle(e \mathrm{~b})$} \\
\cline { 2 - 5 } & & \multicolumn{3}{c}{ Previous measurements } \\
\cline { 2 - 5 } & & \multicolumn{1}{c}{$[8]$} & {$[11]$} & {$[9]$} \\
\hline $0_{1}^{+} \rightarrow 2_{1}^{+}$ & $0.68_{-0.01}^{+0.01}$ & $0.725(18)$ & $0.689(17)$ & $-0.725^{\mathrm{a}}$ \\
$0_{1}^{+} \rightarrow 2_{2}^{+}$ & $0.103_{-0.001}^{+0.002}$ & $0.106(4)$ & $0.089(6)$ & $0.097(4)$ \\
$0_{1}^{+} \rightarrow 2_{3}^{+}$ & $-0.016_{-0.003}^{+0.003}$ & & & $<0.03$ \\
$2_{1}^{+} \rightarrow 0_{2}^{+}$ & $0.513_{-0.004}^{+0.009}$ & $0.436(7)$ & & $-0.425(34)$ \\
$2_{1}^{+} \rightarrow 2_{2}^{+}$ & $0.94_{-0.02}^{+0.02}$ & $0.94(1)$ & $0.83(6)$ & $-0.86(4)$ \\
$2_{1}^{+} \rightarrow 4_{1}^{+}$ & $1.33_{-0.02}^{+0.03}$ & $1.325(1)$ & $1.31(9)$ & $1.38(5)$ \\
$2_{1}^{+} \rightarrow 2_{3}^{+}$ & $-0.070_{-0.006}^{+0.007}$ & & & $0.26(3)$ \\
$2_{1}^{+} \rightarrow 4_{2}^{+}$ & $0.063_{-0.012}^{+0.025}$ & & & \\
$0_{2}^{+} \rightarrow 2_{2}^{+}$ & $-0.32_{-0.02}^{+0.03}$ & & & $<0.1$ \\
$0_{2}^{+} \rightarrow 2_{3}^{+}$ & $0.506_{-0.006}^{+0.008}$ & & & $0.47(5)$ \\
$2_{2}^{+} \rightarrow 4_{1}^{+}$ & $0.77_{-0.10}^{+0.13}$ & & & $0.1(1)$ \\
$2_{2}^{+} \rightarrow 2_{3}^{+}$ & $0.40_{-0.13}^{+0.15}$ & & & $0.3(3)$ \\
$2_{2}^{+} \rightarrow 4_{2}^{+}$ & $1.02_{-0.03}^{+0.04}$ & & & $0.89(7)$ \\
$4_{1}^{+} \rightarrow 2_{3}^{+}$ & $0.83_{-0.04}^{+0.07}$ & & & $-0.5(2)$ \\
$4_{1}^{+} \rightarrow 4_{2}^{+}$ & $0.99_{-0.05}^{+0.05}$ & & & $-0.87(7)$ \\
$4_{1}^{+} \rightarrow 6_{1}^{+}$ & $1.83_{-0.06}^{+0.06}$ & & & $-1.86(13)$ \\
\hline \hline
\end{tabular}

aTaken from Ref. [8]. All signs in Ref. [9] are predicted by the IBM-2 model [10].

is more than $50 \%$ smaller than the one obtained in the present work, and the $\left\langle 2_{1}^{+}\|E 2\| 2_{3}^{+}\right\rangle$matrix element is four times larger. The present analysis shows that the $2_{3}^{+}$state at an energy of $1463 \mathrm{keV}$ is populated in the present experiment with a higher probability via three-step Coulomb excitation within the $\left(0_{1}^{+} \rightarrow 2_{1}^{+}\right) \otimes\left(2_{1}^{+} \rightarrow 4_{1}^{+}\right) \otimes\left(4_{1}^{+} \rightarrow 2_{3}^{+}\right)$pattern than via $\left(0_{1}^{+} \rightarrow 2_{1}^{+}\right) \otimes\left(2_{1}^{+} \rightarrow 2_{3}^{+}\right)$.

A significant discrepancy is observed for the $\left\langle 2_{2}^{+}\|E 2\| 4_{1}^{+}\right\rangle$ matrix element. It should be noted that its previous, much lower value [9] is in better agreement with a quasivibrational character of ${ }^{100} \mathrm{Mo}$, suggested by the energy difference between the $2_{2}^{+}$and $4_{1}^{+}$states being as small as $72 \mathrm{keV}$. The value determined in the present work is suprisingly large compared to the predictions of the vibrational model

TABLE V. Reduced diagonal E2 matrix elements in ${ }^{100} \mathrm{Mo}$ obtained in the present work, compared to the $E 2$ matrix elements determined previously (Refs. $[9,13,14]$ ).

\begin{tabular}{lrrrr}
\hline \hline$I_{i} \rightarrow I_{i}$ & \multicolumn{4}{c}{$\left\langle I_{i}\|E 2\| I_{i}\right\rangle(e \mathrm{~b})$} \\
\cline { 2 - 5 } & & \multicolumn{3}{c}{ Previous measurements } \\
\cline { 2 - 5 } & & {$[13]$} & {$[14]$} & {$[9]$} \\
\hline $2_{1}^{+} \rightarrow 2_{1}^{+}$ & $-0.33_{-0.10}^{+0.10}$ & $-0.55(12)$ & $-0.51(10)$ & $-0.4(3)$ \\
$2_{2}^{+} \rightarrow 2_{2}^{+}$ & $1.20_{-0.08}^{+0.10}$ & & $-0.9(6)$ \\
$2_{3}^{+} \rightarrow 2_{3}^{+}$ & $-0.24_{-0.07}^{+0.12}$ & & \\
$4_{1}^{+} \rightarrow 4_{1}^{+}$ & $-0.35_{-0.18}^{+0.18}$ & & $-0.7(4)$ \\
\hline \hline
\end{tabular}


TABLE VI. $M 1$ matrix elements in ${ }^{100}$ Mo obtained in the present work, compared to the ones determined on the basis of previous experiments [9,24-27]. The values listed in the second column were calculated from evaluated $B(M 1)$ values [28] assuming a positive sign.

\begin{tabular}{lcc}
\hline \hline$I_{i} \rightarrow I_{f}$ & \multicolumn{2}{c}{$\left\langle I_{i}\|M 1\| I_{f}\right\rangle\left(\mu_{n}\right)$} \\
\cline { 2 - 3 } & Present work & Previous measurements \\
\hline $2_{1}^{+} \rightarrow 2_{2}^{+}$ & $0.08_{-0.03}^{+0.03}$ & $0.085(22)$ \\
$2_{1}^{+} \rightarrow 2_{3}^{+}$ & $0.202_{-0.003}^{+0.003}$ & $0.177(22)$ \\
\hline \hline
\end{tabular}

(where transitions within the phonon multiplets are forbidden) suggesting that low-lying collective states of ${ }^{100}$ Mo cannot be easily interpreted.

Collected experimental data were sufficient and precise enough to determine four diagonal $E 2$ matrix elements: those of all observed $2^{+}$states as well as of the $4_{1}^{+}$state. The diagonal matrix element $\left\langle 2_{3}^{+}\|E 2\| 2_{3}^{+}\right\rangle$was extracted in this work. The results for $2_{1}^{+}, 2_{2}^{+}$, and $4_{1}^{+}$states were compared with the ones obtained in Refs. $[9,13,14]$. All previous measurements indicate large values of the diagonal E2 matrix elements; however, some of them were extracted with relative errors as high as $75 \%$ [9]. The diagonal matrix element of the $2_{1}^{+}$ state was previously measured in Coulomb excitation [13,14]. In both experiments a ${ }^{100}$ Mo target was bombarded with ${ }^{4} \mathrm{He}$ and ${ }^{16} \mathrm{O}$ beams in order to measure the excitation probability of the $2_{1}^{+}$state that depends on the quadrupole moment of this state via the reorientation effect. Ratios of elastically and inelastically scattered beam particles were compared in Ref. [13], while in the experiment of Naqib et al. [14] the $2_{1}^{+} \rightarrow 0_{1}^{+} \gamma$-ray transition was observed in coincidence with scattered ${ }^{4} \mathrm{He}$ and ${ }^{16} \mathrm{O}$ ions. The $2_{1}^{+} \rightarrow 0_{1}^{+}$transition yields were measured for each beam and normalized to the number of the elastically scattered projectiles, and from the ratio of the two normalized yields the quadrupole moment was extracted. In the calculations all other matrix elements in ${ }^{100}$ Mo were adopted after Ref. [8].

The data analysis presented in Ref. [14] aiming at the extraction of the diagonal matrix element of the $2_{1}^{+}$state is questionable and does not lead to unequivocal conclusions. We performed a simulation using the GOSIA code in order to calculate the normalized $\gamma$-ray intensity ratio of the $2_{1}^{+} \rightarrow 0_{1}^{+}$ transition measured in coincidence with ${ }^{4} \mathrm{He}$ and ${ }^{16} \mathrm{O}$ ions, as defined in Ref. [14] but assuming the set of $E 2$ matrix

TABLE VII. Absolute values of reduced $E 3$ matrix elements in ${ }^{100}$ Mo obtained in the present work, compared to the $E 3$ matrix elements determined previously [9] and the value calculated from the $B(E 3)[8]$ assuming a positive sign.

\begin{tabular}{llll}
\hline \hline$I_{i} \rightarrow I_{f}$ & \multicolumn{3}{c}{$\left|\left\langle I_{i}\|E 3\| I_{f}\right\rangle\right|\left(e \mathrm{~b}^{3 / 2}\right)$} \\
\cline { 2 - 4 } & Present work & \multicolumn{2}{c}{ Previous measurements } \\
\hline $0_{1}^{+} \rightarrow 3^{-}$ & $0.44_{-0.01}^{+0.01}$ & $0.36(2)$ & $0.48(8)$ \\
$2_{1}^{+} \rightarrow 3^{-}$ & $0.33_{-0.04}^{+0.03}$ & & \\
\hline \hline
\end{tabular}

TABLE VIII. Absolute values of reduced $E 1$ matrix elements in ${ }^{100}$ Mo obtained in the present work, compared to the ones determined previously in Ref. [9].

\begin{tabular}{llc}
\hline \hline$I_{i} \rightarrow I_{f}$ & \multicolumn{2}{c}{$\left|\left\langle I_{i}\|E 1\| I_{f}\right\rangle\right|\left(\times 10^{-2} e \mathrm{~b}^{1 / 2}\right)$} \\
\cline { 2 - 3 } & Present work & Previous measurements [9] \\
\hline $2_{1}^{+} \rightarrow 3^{-}$ & $0.052_{-0.005}^{+0.006}$ & $0.051(6)$ \\
$2_{2}^{+} \rightarrow 3^{-}$ & $0.16_{-0.01}^{+0.02}$ & $0.17(2)$ \\
\hline \hline
\end{tabular}

elements derived in the present work (Table I). The resulting intensity ratio deviated by $0.2 \sigma$ only from the experimental value published by Naqib et al. [14].

The excitation probability of the $2_{1}^{+}$state depends on the sign of the interference term,

$$
P_{3}=\left\langle 2_{2}^{+}\|E 2\| 0_{1}^{+}\right\rangle\left\langle 2_{2}^{+}\|E 2\| 2_{1}^{+}\right\rangle\left\langle 2_{1}^{+}\|E 2\| 0_{1}^{+}\right\rangle
$$

As relative phases of the relevant matrix elements taken from Ref. [8] were not known, two alternative solutions for the $\left\langle 2_{1}^{+}\|E 2\| 2_{1}^{+}\right\rangle$matrix element were reported in Ref. [14]. One of them corresponds to the constructive interefence (positive sign of the $P_{3}$ value) and the other to the destructive one (negative sign of the $P_{3}$ value). The sign of the $P_{3}$ term obtained in the present work is positive and, accordingly, in Table $\mathrm{V}$ previously measured diagonal matrix elements of the $2_{1}^{+}$state $[9,13,14]$ are those which result from constructive interference.

In the current experiment we observed the population of the $3^{-}$state by measuring its decay to the $2_{2}^{+}$state via $\gamma$-ray transition of $844 \mathrm{keV}$ energy. In low-energy Coulomb excitation $E 1$ couplings have negligible influence on the excitation process and, in consequence, an opposite-parity state is populated almost entirely via $E 3$ coupling. However, $E 3$ transitions cannot compete with $E 1$ 's during de-excitation of such a state. This leads to a possible experimental sensitivity to both $E 1$ and $E 3$ matrix elements.

Taking into account known spectroscopic data-branching ratios $\left(3^{-} \rightarrow 0_{1}^{+}\right) /\left(3^{-} \rightarrow 2_{2}^{+}\right)$and $\left(3^{-} \rightarrow 2_{1}^{+}\right) /\left(3^{-} \rightarrow 2_{2}^{+}\right)$ [7,8,24-26], as well as the lifetime of this state [9]—it was possible to extract absolute values of $E 3$ and $E 1$ matrix elements presented in Tables VII and VIII.

Similar values of both E3 matrix elements linking $3^{-}$ to positive-parity states indicate that the two-step Coulomb excitation of the $3^{-}$state, $\left(0_{1}^{+} \rightarrow 2_{1}^{+}\right) \otimes\left(2_{1}^{+} \rightarrow 3^{-}\right)$has to be considered as a viable excitation path. This was not taken into account in previous Coulomb excitation studies of ${ }^{100} \mathrm{Mo}$.

\section{MODEL-INDEPENDENT DETERMINATION OF QUADRUPOLE DEFORMATION PARAMETERS}

The set of E2 reduced matrix elements can be analyzed using a nuclear model-independent description of the nuclear shape in terms of quadrupole deformation parameters.

The quadrupole sum rules method [31,32] is based on the fact that the electric multipole transition operator $E(\lambda, \mu)$ is a spherical tensor and thus its zero-coupled products can be formed, which are rotationally invariant. Such rotational invariants can be linked to deformation parameters in the intrinsic frame of the nucleus. The lowest-order 
rotational-invariant products of the $E 2$ operator provide information on the quadrupole deformation parameters: the overall deformation $(Q)$ and triaxiality $(\delta)$. The $Q$ and $\delta$ parameters are analogous to Bohr's $\beta$ and $\gamma$ parameters, but describe the charge rather than the mass distribution.

The basic quadrupole invariant operator is formed from the electric quadrupole tensor $E 2$ in the following way:

$$
\frac{\left\langle Q^{2}\right\rangle}{\sqrt{5}}=\left\langle i\left|[E 2 \times E 2]_{0}\right| i\right\rangle .
$$

On the other hand, one can express the expectation value of the lowest-order product of the $E 2$ operator in the laboratory frame thanks to the expansion over all possible intermediate states using Wigner's $6 j$ symbols:

$$
\begin{aligned}
& \left\langle i\left|[E 2 \times E 2]_{0}\right| i\right\rangle \\
& \quad=\frac{(-1)^{2 I_{i}}}{\sqrt{\left(2 I_{i}+1\right)}} \sum_{t}\langle i\|\mathrm{E} 2\| t\rangle\langle t\|\mathrm{E} 2\| i\rangle\left\{\begin{array}{ccc}
2 & 2 & 0 \\
I_{i} & I_{i} & I_{t}
\end{array}\right\} .
\end{aligned}
$$

To get information on triaxiality, a higher-order invariant needs to be formed:

$$
\left.\sqrt{\frac{2}{35}}\left\langle Q^{3} \cos (3 \delta)\right\rangle=\langle i|[E 2 \times E 2]_{2} \times E 2\right]_{0}|i\rangle
$$

A similar evaluation using the intermediate state expansion formula yields

$$
\begin{aligned}
& \left.\langle i|[E 2 \times E 2]_{2} \times E 2\right]_{0}|i\rangle \\
& =\frac{(-1)^{2 I_{i}}}{\left(2 I_{i}+1\right)} \sum_{t, u}\langle i\|\mathrm{E} 2\| u\rangle\langle u\|\mathrm{E} 2\| t\rangle\langle t\|\mathrm{E} 2\| i\rangle\left\{\begin{array}{ccc}
2 & 2 & 2 \\
I_{i} & I_{t} & I_{u}
\end{array}\right\} .
\end{aligned}
$$

In the present paper the quadrupole asymmetry, i.e., the measure of deviation from axial symmetry, is defined as in Ref. [33] under the assumption

$$
\left\langle Q^{3} \cos (3 \delta)\right\rangle \cong\left(\left\langle Q^{2}\right\rangle\right)^{3 / 2}\langle\cos (3 \delta)\rangle
$$

Sums over intermediate states in Eqs. (4) and (6), which formally extend over all states of the system that may be reached by a single $E 2$ transition from the state $i$ or $u$, are in practice limited to the significant $E 2$ matrix elements. The number of relevant matrix elements depends on the degree to which nuclear properties are correlated by collective degrees of freedom.

While the determination of the shape parameters from the measured matrix elements based on the rotational invariants is straightforward, the errors estimation of the latter magnitudes is difficult due to the cross-correlation of the matrix elements. In order to include the cross-correlation effects while calculating errors of the invariants, the same procedure that was used to estimate uncertainties of individual matrix elements (see Sec. IV A) was applied in the present paper.

The quadrupole sum rules method is particularly useful for attributing shape parameters to low-lying $0^{+}$states, whose deformation cannot be directly inferred from, e.g., a quadrupole moment measurement.

\section{A. Quadrupole shape parameters of ${ }^{100} \mathrm{Mo}$}

The set of reduced E2 matrix elements extracted in the present work was rich and precise enough to determine shape parameters of the two lowest $0^{+}$states in ${ }^{100} \mathrm{Mo}$.

The main contributions of the products of various experimental matrix elements to the values of the $\left\langle Q^{2}\right\rangle$ invariants obtained for the ground and excited $0^{+}$states in the ${ }^{100} \mathrm{Mo}$ isotope are presented in Table IX.

One can notice that the average overall deformation $\left\langle Q^{2}\right\rangle$ of the ${ }^{100} \mathrm{Mo}$ nucleus in its $0^{+}$ground state is almost completely defined by the magnitude of the $\left\langle 0_{1}^{+}\|E 2\| 2_{1}^{+}\right\rangle$ matrix element; i.e., similarly to what is observed for well deformed nuclei, most of the $E 2$ strength is exhausted by the first $2^{+}$state. In contrast, when determining the overall deformation for the excited $0_{2}^{+}$state, one needs to take into account the contributions resulting from coupling of this state to all three $2^{+}$states excited in the experiment. The non-negligible contribution from higher-lying $2^{+}$states to the overall deformation parameter of the $0_{2}^{+}$state might be caused by the shape coexistence and configuration mixing of both $0^{+}$ states.

To obtain the triaxiality parameter, $\langle\cos (3 \delta)\rangle$, more experimental information is needed than in the case of the $\left\langle Q^{2}\right\rangle$ value. According to Eq. (6) the triaxiality parameter for low-lying $0^{+}$states can be determined from a triple product $\left[[E 2 \times E 2]_{2} \times E 2\right]_{0}$. As shown in Table X, larger sets of matrix elements are necessary to calculate the $\left\langle Q^{3} \cos (3 \delta)\right\rangle$ invariant, including not only the transitional ones that couple all experimentally observed $0^{+}$and $2^{+}$states via a single $E 2$ transition, but also diagonal E2 matrix elements of all excited $2^{+}$states. Moreover, relative signs of all relevant matrix elements are essential.

It should be noted (Table X) that, to deduce the quadrupole asymmetry of ${ }^{100} \mathrm{Mo}$ in its ground state, the experimental information on the diagonal $E 2$ matrix element of the $2_{1}^{+}$ state is not sufficient. The $E 2 \times E 2 \times E 2$ loops involving $E 2$ matrix elements related to the $2_{2}^{+}$state are almost equally important, and, as their signs are opposite to the one including the $\left\langle 2_{1}^{+}\|E 2\| 2_{1}^{+}\right\rangle$matrix element, these components almost cancel out, resulting in the value of $\left\langle 0_{1}^{+}\left|Q^{3} \cos (3 \delta)\right| 0_{1}^{+}\right\rangle$being close to zero.

TABLE IX. Contribution of individual matrix elements to the values of the $\left\langle 0_{1}^{+}\left|Q^{2}\right| 0_{1}^{+}\right\rangle$and $\left\langle 0_{2}^{+}\left|Q^{2}\right| 0_{2}^{+}\right\rangle$invariants in ${ }^{100} \mathrm{Mo}$. The $\sqrt{5} \times\left\{\begin{array}{lll}2 & 2 & 0 \\ 0 & 0 & 2\end{array}\right\}$ factor, multiplying the contributions according to Eqs. (3) and (4), is in this case equal to 1.

\begin{tabular}{ccc}
\hline \hline State & $\begin{array}{c}\text { Component } \\
E 2 \times E 2\end{array}$ & $\begin{array}{c}\text { Contribution to } \\
\left\langle Q^{2}\right\rangle\left(e^{2} \mathrm{~b}^{2}\right)\end{array}$ \\
\hline \multirow{4}{*}{$0_{1}^{+}$} & $\left\langle 0_{1}^{+}\|E 2\| 2_{1}^{+}\right\rangle\left\langle 2_{1}^{+}\|E 2\| 0_{1}^{+}\right\rangle$ & 0.46 \\
& $\left\langle 0_{1}^{+}\|E 2\| 2_{2}^{+}\right\rangle\left\langle 2_{2}^{+}\|E 2\| 0_{1}^{+}\right\rangle$ & 0.01 \\
& $\left\langle 0_{1}^{+}\|E 2\| 2_{3}^{+}\right\rangle\left\langle 2_{3}^{+}\|E 2\| 0_{1}^{+}\right\rangle$ & 0.0002 \\
& $\left\langle 0_{1}^{+}\left|Q^{2}\right| 0_{1}^{+}\right\rangle$ & $0.47(3)$ \\
$0_{2}^{+}$ & $\left\langle 0_{2}^{+}\|E 2\| 2_{1}^{+}\right\rangle\left\langle 2_{1}^{+}\|E 2\| 0_{2}^{+}\right\rangle$ & 0.26 \\
& $\left\langle 0_{2}^{+}\|E 2\| 2_{2}^{+}\right\rangle\left\langle 2_{2}^{+}\|E 2\| 0_{2}^{+}\right\rangle$ & 0.10 \\
& $\left\langle 0_{2}^{+}\|E 2\| 2_{3}^{+}\right\rangle\left\langle 2_{3}^{+}\|E 2\| 0_{2}^{+}\right\rangle$ & 0.25 \\
& $\left\langle 0_{2}^{+}\left|Q^{2}\right| 0_{2}^{+}\right\rangle$ & $0.62(3)$ \\
\hline
\end{tabular}


TABLE X. Contribution of individual matrix elements to the values of the $\left\langle 0_{1}^{+}\left|Q^{3} \cos (3 \delta)\right| 0_{1}^{+}\right\rangle$and $\left\langle 0_{2}^{+}\left|Q^{3} \cos (3 \delta)\right| 0_{2}^{+}\right\rangle$invariants in ${ }^{100} \mathrm{Mo}$. Presented invariants, accordingly to Eqs. (5) and (6), result from the multiplication of the sum of all contributions by the factor $(-1) \times \sqrt{\frac{35}{2}} \times\left\{\begin{array}{lll}2 & 2 & 2 \\ 0 & 2 & 2\end{array}\right\}$, equal to -0.837 .

\begin{tabular}{ccc}
\hline \hline State & Component & Contribution to \\
$E 2 \times E 2 \times E 2$ & $\left\langle Q^{3} \cos 3 \delta\right\rangle\left(e^{3} \mathrm{~b}^{3}\right)$ \\
\hline & $\left\langle 0_{1}^{+}\|E 2\| 2_{1}^{+}\right\rangle\left\langle 2_{1}^{+}\|E 2\| 2_{1}^{+}\right\rangle\left\langle 2_{1}^{+}\|E 2\| 0_{1}^{+}\right\rangle$ & -0.155 \\
$\left\langle 0_{1}^{+}\|E 2\| 2_{1}^{+}\right\rangle\left\langle 2_{1}^{+}\|E 2\| 2_{2}^{+}\right\rangle\left\langle 2_{2}^{+}\|E 2\| 0_{1}^{+}\right\rangle$ & 0.132 \\
& $\left\langle 0_{1}^{+}\|E 2\| 2_{1}^{+}\right\rangle\left\langle 2_{1}^{+}\|E 2\| 2_{3}^{+}\right\rangle\left\langle 2_{3}^{+}\|E 2\| 0_{1}^{+}\right\rangle$ & 0.002 \\
$0_{1}^{+} \quad\left\langle 0_{1}^{+}\|E 2\| 2_{2}^{+}\right\rangle\left\langle 2_{2}^{+}\|E 2\| 2_{2}^{+}\right\rangle\left\langle 2_{2}^{+}\|E 2\| 0_{1}^{+}\right\rangle$ & 0.013 \\
& $\left\langle 0_{1}^{+}\|E 2\| 2_{2}^{+}\right\rangle\left\langle 2_{2}^{+}\|E 2\| 2_{3}^{+}\right\rangle\left\langle 2_{3}^{+}\|E 2\| 0_{1}^{+}\right\rangle$ & -0.001 \\
$\left\langle 0_{1}^{+}\|E 2\| 2_{3}^{+}\right\rangle\left\langle 2_{3}^{+}\|E 2\| 2_{3}^{+}\right\rangle\left\langle 2_{3}^{+}\|E 2\| 0_{1}^{+}\right\rangle$ & -0.0001 \\
& Sum of all contributions & -0.009 \\
$\left\langle 0_{1}^{+}\left|Q^{3} \cos (3 \delta)\right| 0_{1}^{+}\right\rangle$ & $0.01(6)$ \\
& $\left\langle 0_{2}^{+}\|E 2\| 2_{1}^{+}\right\rangle\left\langle 2_{1}^{+}\|E 2\| 2_{1}^{+}\right\rangle\left\langle 0_{2}^{+}\|E 2\| 2_{1}^{+}\right\rangle$ & -0.09 \\
$\left\langle 0_{2}^{+}\|E 2\| 2_{1}^{+}\right\rangle\left\langle 2_{1}^{+}\|E 2\| 2_{2}^{+}\right\rangle\left\langle 2_{2}^{+}\|E 2\| 0_{2}^{+}\right\rangle$ & -0.31 \\
$\left\langle 0_{2}^{+}\|E 2\| 2_{1}^{+}\right\rangle\left\langle 2_{1}^{+}\|E 2\| 2_{3}^{+}\right\rangle\left\langle 2_{3}^{+}\|E 2\| 0_{2}^{+}\right\rangle$ & -0.04 \\
$\left\langle 0_{2}^{+}\|E 2\| 2_{2}^{+}\right\rangle\left\langle 2_{2}^{+}\|E 2\| 2_{2}^{+}\right\rangle\left\langle 2_{2}^{+}\|E 2\| 0_{2}^{+}\right\rangle$ & 0.12 \\
$\left\langle 0_{2}^{+}\|E 2\| 2_{2}^{+}\right\rangle\left\langle 2_{2}^{+}\|E 2\| 2_{3}^{+}\right\rangle\left\langle 2_{3}^{+}\|E 2\| 0_{2}^{+}\right\rangle$ & -0.13 \\
$\left\langle 0_{2}^{+}\|E 2\| 2_{3}^{+}\right\rangle\left\langle 2_{3}^{+}\|E 2\| 2_{3}^{+}\right\rangle\left\langle 2_{3}^{+}\|E 2\| 0_{2}^{+}\right\rangle$ & -0.06 \\
Sum of all contributions & -0.51 \\
$\left\langle 0_{2}^{+}\left|Q^{3} \cos (3 \delta)\right| 0_{2}^{+}\right\rangle$ & $0.42(6)$ \\
\hline \hline
\end{tabular}

To determine the triaxiality for the excited $0_{2}^{+}$state in ${ }^{100} \mathrm{Mo}$, a different set of the $E 2$ matrix elements than in case of the ground state needs to be known, and more matrix elements have a significant influence on the final result. The $E 2$ couplings between the $0_{2}^{+}$state and all observed excited $2^{+}$states are important in this case, as are the diagonal matrix elements of the $2_{1}^{+}$and $2_{2}^{+}$states.

The resulting quadrupole deformation parameters of ${ }^{100} \mathrm{Mo}$ in its first and second $0^{+}$states are presented in Fig. 8. Results are compared to the values obtained for the neighboring isotopes ${ }^{96} \mathrm{Mo} \mathrm{[34]} \mathrm{and}{ }^{98} \mathrm{Mo} \mathrm{[4]}$. The average overall deformation of both $0^{+}$states in ${ }^{100} \mathrm{Mo}$ is noticeably larger than in the ${ }^{96,98}$ Mo isotopes.

The obtained expectation values of $Q^{2}$ indicate that the ${ }^{100}$ Mo nucleus is less deformed in the ground state than in the excited $\mathrm{O}_{2}^{+}$state. This trend is inverse to what is observed for the ${ }^{96} \mathrm{Mo}$ isotope: the ground state in ${ }^{96} \mathrm{Mo}$ is deformed, while the excited $0_{2}^{+}$state is almost spherical. The overall deformation in ${ }^{98} \mathrm{Mo}$ is equal within uncertainty limits for both $0^{+}$states [4].

The expectation values of $\cos (3 \delta)$ indicate almost purely prolate shapes for the excited $0^{+}$states of ${ }^{100} \mathrm{Mo}$ and ${ }^{98} \mathrm{Mo}$ [4]. As the shape of the $0_{2}^{+}$state in ${ }^{96} \mathrm{Mo}$ is close to spherical, the triaxiality parameter for this state was obtained with a large error [34] and this data point is not plotted in Fig. 13. The expectation values of $\cos (3 \delta)$ were found to be consistent with zero within the error bars for the ground states of all molybdenum isotopes under study, indicating their triaxial deformation. It should be mentioned that the expectation value of $\cos (3 \delta)=0\left(\delta=30^{\circ}\right)$ has a meaning of maximum triaxiality in the case of rigid, well defined shapes. Shapes discussed in the present work are rather diffused and
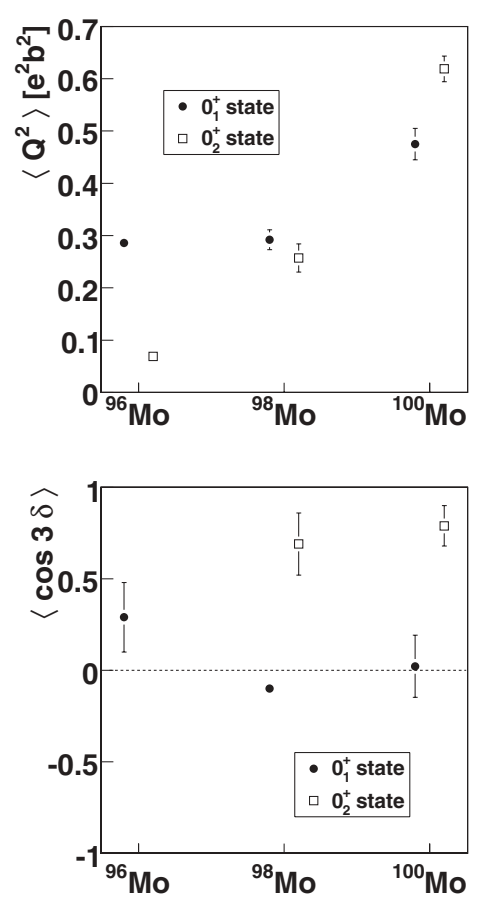

FIG. 8. Experimental expectation values of $Q^{2}$ (quadrupole overall deformation parameter) and $\cos (3 \delta)$ (quadrupole asymmetry parameter) for the two first $0^{+}$states in ${ }^{96,98,100}$ Mo nuclei; $\langle\cos (3 \delta)\rangle=$ 1 corresponds to the prolate shape, $\langle\cos (3 \delta)\rangle=-1$ to oblate shape, and $\langle\cos (3 \delta)\rangle=0$ to triaxiality. The overall deformation of ${ }^{100} \mathrm{Mo}$ in both $0^{+}$states is larger compared to the results obtained for ${ }^{96} \mathrm{Mo}$ and ${ }^{98} \mathrm{Mo}$. The asymetry parameter indicates the triaxial shape of the $0_{1}^{+}$ state and a prolate shape of the excited $0_{2}^{+}$state in ${ }^{98,100} \mathrm{Mo}$.

the obtained deformation parameters should be considered as average ones. In particular, $\cos (3 \delta)=0$ may correspond to a situation where the probability density distribution is symmetrical around $\delta$ equal to $30^{\circ}$ but does not necessarily have a minimum at this point.

\section{COMPARISON WITH THEORY AND DISCUSSION}

The present results represent the first direct measurements of the nuclear shape for the ground and excited $0^{+}$states in the ${ }^{100} \mathrm{Mo}$ isotope. In the following the experimental results are compared to calculations of the quadrupole collective model based on the general Bohr Hamiltonian (GBH). Experimental and theoretical values for excitation energies, matrix elements, and quadrupole deformation parameters are discussed and summarized in Tables XI and XII and presented in Figs. 12 and 13 .

\section{A. General Bohr Hamiltonian}

In this section we present a theoretical model which has been applied to describe electromagnetic properties of the 100 Mo nucleus that were a subject of the discussion of the previous sections. The main theoretical tool is the general Bohr Hamiltonian, which has an advantage of acting in the full space of quadrupole degrees of freedom. Such a model that treats simultaneously and on equal footing both vibrational and rotational excitations is especially important 
for transitional nuclei such as ${ }^{100} \mathrm{Mo}$. The general Bohr Hamiltonian is determined by seven functions (potential energy and six inertial functions which enter the kinetic part) which are obtained from the microscopic theory employing effective nucleon-nucleon interactions of the Skyrme type. The connection between a level of nucleon degrees of freedom and collective phenomena is built by using the adiabatic time-dependent Hartree-Fock-Bogoliubov (ATDHFB) theory; see the recent review [35] and references therein for more details. Here we recall only some main points.

First we specify collective variables which we use to describe quadrupole excitations. The quadrupole variables have to form a second rank tensor with respect to the $\mathrm{SO}(3)$ rotation group and have to be of positive parity. There are several ways of choosing such variables. The oldest and probably best known one starts with expanding a shape of a nucleus in a series of spherical harmonics $R(\theta, \phi)=$ $R_{0}\left(1+\sum_{\mu} \alpha_{2 \mu} Y_{2 m}^{*}\right)[36,37]$. Another possibility is to define the quadrupole variables through parameters of ellipsoids of equipotential surfaces of a phenomenological one-particle potential (e.g., of the Nilsson or Woods-Saxon type) [38-40]. Here we choose the collective variables as proportional to components of the quadrupole mass tensor (with no a priori assumption about the shape of a nucleus):

$$
\alpha_{2 \mu} \sim\left\langle\Phi\left|Q_{2 \mu}^{\text {(mass) }}\right| \Phi\right\rangle,
$$

where

$$
Q_{2 \mu}^{\text {(mass) }}=\sum_{i=1}^{A} r_{i}^{2} Y_{2 \mu}\left(\theta_{i}, \phi_{i}\right)
$$

and $\Phi$ is a microscopic nuclear wave function. Such a definition is natural and is widely adopted within self-consistent mean field theories. The mean field is obtained by using effective interactions of the Skyrme [41] or the Gogny type $[42,43]$ or in the relativistic framework (RMF) [44].

The intrinsic (also called principal axes or body-fixed) frame of reference is determined by the conditions $\alpha_{2 \pm 1}^{\text {(int) }}=$ $0, \alpha_{22}^{\text {(int) }}=\alpha_{2-2}^{\text {(int) }}$. Thus the quadrupole tensor $\alpha_{2 \mu}$ can be equivalently described by two variables $\alpha_{20}^{\text {(int) }}, \alpha_{22}^{\text {(int) }}$ which describe nuclear deformation and three Euler angles $\Omega$ which give orientation of the intrinsic frame with respect to the fixed (laboratory) frame. In the intrinsic frame, instead of $Q_{2 \mu}^{\text {(mass) }}$, one often uses the $Q_{0}, Q_{2}$ operators:

$$
\begin{aligned}
& Q_{0}^{\text {(mass) }}=\sum_{i=1}^{A}\left(3 z_{i}^{2}-r_{i}^{2}\right)=\sqrt{16 \pi / 5} Q_{20}^{\text {(mass) }}, \\
& Q_{2}^{\text {(mass) }}=\sum_{i=1}^{A} \sqrt{3}\left(x_{i}^{2}-y_{i}^{2}\right) .
\end{aligned}
$$

Finally, the $\beta, \gamma$ deformation variables are defined by the relations

$$
\begin{aligned}
& \beta \cos \gamma=\alpha_{20}^{\text {(int) }}=c\left\langle\Phi\left|Q_{0}^{(\text {mass })}\right| \Phi\right\rangle, \\
& \beta \sin \gamma=\sqrt{2} \alpha_{22}^{\text {(int) }}=c\left\langle\Phi\left|Q_{2}^{\text {(mass) }}\right| \Phi\right\rangle,
\end{aligned}
$$

where the coefficient $c$ is conventionally chosen as

$$
c=\sqrt{\pi / 5} / A \overline{r^{2}}
$$

with the liquid drop model estimation of the mean square radius $\overline{r^{2}}=3\left(r_{0} A^{1 / 3}\right)^{2} / 5, r_{0}=1.2 \mathrm{fm}$. In the following we use almost exclusively the intrinsic frame and $\beta, \gamma$ variables.

Under some natural assumptions (see, e.g., Ref. [35]) the general quantum Hamiltonian in the quadrupole collective space (in other words the general Bohr Hamiltonian) can be written in the form

$$
\begin{aligned}
H= & T_{\text {vib }}+T_{\text {rot }}+V \\
T_{\text {vib }}= & -\frac{\hbar^{2}}{2 \sqrt{w r}}\left\{\frac { 1 } { \beta ^ { 4 } } \left[\partial_{\beta}\left(\beta^{4} \sqrt{\frac{r}{w}} B_{\gamma \gamma}(\beta, \gamma) \partial_{\beta}\right)\right.\right. \\
& \left.-\partial_{\beta}\left(\beta^{3} \sqrt{\frac{r}{w}} B_{\beta \gamma}(\beta, \gamma) \partial_{\gamma}\right)\right] \\
& +\frac{1}{\beta \sin 3 \gamma}\left[-\partial_{\gamma}\left(\sqrt{\frac{r}{w}} \sin 3 \gamma B_{\beta \gamma}(\beta, \gamma) \partial_{\beta}\right)\right. \\
& \left.\left.+\frac{1}{\beta} \partial_{\gamma}\left(\sqrt{\frac{r}{w}} \sin 3 \gamma B_{\beta \beta}(\beta, \gamma) \partial_{\gamma}\right)\right]\right\} \\
T_{\text {rot }}= & \frac{1}{2} \sum_{k=1}^{3} I_{k}^{2} / J_{k}, \quad J_{k}=4 \beta^{2} B_{k}(\beta, \gamma) \sin ^{2}(\gamma-2 k \pi / 3)
\end{aligned}
$$

$$
V=V(\beta, \gamma),
$$

and where

$$
w=B_{\beta \beta} B_{\gamma \gamma}-B_{\beta \gamma}^{2}, \quad r=B_{1} B_{2} B_{3} .
$$

In the formula (15) the operators $I_{k}, k=1,2,3$ are components of the angular momentum in the intrinsic frame. The functions $B_{\beta \beta}, B_{\beta \gamma}, B_{\gamma \gamma}, B_{k}$ depend on deformation variables and are called mass parameters or, more precisely, inertial functions, while $J_{k}$ are the moments of inertia. A volume element in the collective space is

$$
d \tau_{\text {coll }}=2^{3} \sqrt{w r} \beta^{4}|\sin 3 \gamma| d \beta d \gamma d \Omega,
$$

where $d \Omega$ is the volume element in the Euler angle space. The physical meaning of the vibrational part the Hamiltonian (14) can probably be seen more easily by looking at its classical counterpart [37,45].

There are two main approaches to determine the seven functions (mass parameters plus potential energy) which enter the general Bohr Hamiltonian. One approach consists of assuming a reasonable form of these functions with some free parameters which are fixed by comparing with experimental collective properties. Examples of such scheme are provided by the general collective model [46] and more recent papers on the so-called dynamical symmetries inspired by the interacting boson model [47]. We use the other method which aims at calculating these functions starting from a microscopic theory, i.e., dealing with nucleon degrees of freedom. In this approach we do not introduce any additional free parameters and the prediction of collective properties is based solely on the knowledge of effective nucleon-nucleon interactions. The 
inertial functions and potential energy are obtained in the framework of the ATDHFB theory, which is closely related to the cranking concept and can be traced back to the seminal papers [48,49] (see also Ref. [50]).

In the ATDHFB calculations we need a set of the BCS-type functions $\Phi$ which are parametrized by the collective variables $\beta, \gamma$. This set is obtained by self-consistent calculations with the constraints on the expectation values of the $Q_{0}^{\text {(mass) }}, Q_{2}^{\text {(mass) }}$ operators:

$$
\delta\left\langle\Phi\left|H^{(\text {micr })}-\lambda_{0} Q_{0}^{\text {(mass) }}-\lambda_{2} Q_{2}^{\text {(mass) }}\right| \Phi\right\rangle=0
$$

with

$$
\left\langle\Phi\left|Q_{0}^{\text {(mass) }}\right| \Phi\right\rangle=q_{0}^{(\mathrm{m})}, \quad\left\langle\Phi\left|Q_{2}^{(\text {mass })}\right| \Phi\right\rangle=q_{2}^{(\mathrm{m})} .
$$

Here $H^{(\mathrm{micr})}$ is a nuclear microscopic many-body Hamiltonian. We assume that the states $\Phi$ are invariant against the $D_{2 h}$ group (generated by rotations by the $\pi$ angle around three perpendicular axes and inversion) in order to fulfill conditions determining the intrinsic frame.

The cranking approximation of the ATDHFB method, which we use in our caculations, neglects the effects of a time-odd component of the mean field induced by a collective motion (sometimes called the Thouless-Valatin term). Due to serious technical difficulties such effects are taken into account only approximately by multiplying the inertial functions (or only moments of inertia) by a constant factor typically in the range 1.2-1.4; see Refs. [41-44].

The collective operator for $E 2$ transitions is defined as

$$
q_{2 \mu}^{\text {(charge) }}=\left\langle\Phi\left|e \sum_{i=1}^{Z} r_{i}^{2} Y_{2 \mu}\left(\theta_{i}, \phi_{i}\right)\right| \Phi\right\rangle .
$$

With good accuracy we have $q_{2 \mu}^{(\mathrm{ch})}=e Z / A Q_{2 \mu}^{\text {(mass) }}$ so the intrinsic frame for the charge quadrupole tensor is the same as for the mass tensor. In the intrinsic frame the invariants discussed in Sec. IV can be expressed as

$$
Q^{2}=q_{20}^{(\mathrm{ch}) 2}+2 q_{22}^{(\mathrm{ch}) 2}=\left(\frac{3 Z R_{0}^{2}}{4 \pi}\right)^{2} \beta^{2}
$$

and

$$
Q^{3} \cos 3 \delta=q_{20}^{(\mathrm{ch})}\left(q_{20}^{(\mathrm{ch}) 2}-6 q_{22}^{(\mathrm{ch}) 2}\right)=\left(\frac{3 Z R_{0}^{2}}{4 \pi}\right)^{3} \beta^{3} \cos 3 \gamma
$$

where $R_{0}=r_{0} A^{1 / 3}$. One should keep in mind that experimentally the nuclear charge distribution in a given state is measured. Connections between the quadrupole invariants and the $\beta, \gamma$ variables depend on the definition of the collective variables. For example, formulas in the Appendix of Ref. [33], which differ from Eqs. (22) and (23), were obtained under the assumption that the deformation variables refer to lengths of semi-axes of a uniformly charged ellipsoid.

\section{B. Results of calculations}

We have performed self-consistent calculations along the lines sketched in the previous subsection with two widely used sets of parameters of the Skyrme interactions, namely SIII [51]

\begin{tabular}{|c|c|c|c|}
\hline \multirow[t]{3}{*}{$I_{i} \rightarrow I_{f}$} & \multicolumn{3}{|c|}{ Transitional matrix elements $\left\langle I_{f}\|E 2\| I_{i}\right\rangle(e \mathrm{~b})$} \\
\hline & \multirow[t]{2}{*}{ Present work } & \multicolumn{2}{|c|}{ GBH model } \\
\hline & & SIII & SLy4 \\
\hline $0_{1}^{+} \rightarrow 2_{1}^{+}$ & $0.68_{-0.01}^{+0.01}$ & 0.77 & 0.64 \\
\hline $0_{1}^{+} \rightarrow 2_{2}^{+}$ & $0.103_{-0.001}^{+0.002}$ & 0.103 & -0.03 \\
\hline $0_{1}^{+} \rightarrow 2_{3}^{+}$ & $-0.016_{-0.003}^{+0.003}$ & 0.05 & 0.01 \\
\hline $2_{1}^{+} \rightarrow 0_{2}^{+}$ & $0.513_{-0.004}^{+0.009}$ & 0.37 & 0.34 \\
\hline $2_{1}^{+} \rightarrow 2_{2}^{+}$ & $0.94_{-0.02}^{+0.02}$ & 0.84 & 0.86 \\
\hline $2_{1}^{+} \rightarrow 4_{1}^{+}$ & $1.33_{-0.02}^{+0.03}$ & 1.37 & 1.16 \\
\hline $2_{1}^{+} \rightarrow 2_{3}^{+}$ & $-0.070_{-0.006}^{+0.007}$ & 0.006 & 0.04 \\
\hline $2_{1}^{+} \rightarrow 4_{2}^{+}$ & $0.063_{-0.012}^{+0.025}$ & 0.108 & 0.034 \\
\hline $2_{1}^{+} \rightarrow 4_{3}^{+}$ & & -0.045 & -0.004 \\
\hline $0_{2}^{+} \rightarrow 2_{2}^{+}$ & $-0.32_{-0.02}^{+0.03}$ & -0.41 & -0.20 \\
\hline $0_{2}^{+} \rightarrow 2_{3}^{+}$ & $0.506_{-0.006}^{+0.008}$ & 0.76 & 0.64 \\
\hline $2_{2}^{+} \rightarrow 4_{1}^{+}$ & $0.77_{-0.10}^{+0.13}$ & -0.23 & -0.19 \\
\hline $2_{2}^{+} \rightarrow 2_{3}^{+}$ & $0.40_{-0.13}^{+0.15}$ & 0.44 & 0.37 \\
\hline $2_{2}^{+} \rightarrow 4_{2}^{+}$ & $1.02_{-0.03}^{+0.04}$ & 1.06 & 0.99 \\
\hline $2_{2}^{+} \rightarrow 4_{3}^{+}$ & & 0.15 & 0.03 \\
\hline $4_{1}^{+} \rightarrow 2_{3}^{+}$ & $0.83_{-0.04}^{+0.07}$ & 0.54 & 0.49 \\
\hline $2_{3}^{+} \rightarrow 4_{2}^{+}$ & & -0.31 & -0.35 \\
\hline $2_{3}^{+} \rightarrow 4_{3}^{+}$ & & -1.04 & -0.20 \\
\hline $4_{1}^{+} \rightarrow 4_{2}^{+}$ & $0.99_{-0.05}^{+0.05}$ & 0.85 & 0.93 \\
\hline $4_{1}^{+} \rightarrow 6_{1}^{+}$ & $1.83_{-0.06}^{+0.06}$ & 1.92 & 1.65 \\
\hline $6_{1}^{+} \rightarrow 8_{1}^{+}$ & & 2.42 & 2.14 \\
\hline \multirow[t]{3}{*}{$I_{i} \rightarrow I_{i}$} & \multicolumn{3}{|c|}{ Diagonal matrix elements $\left\langle I_{i}\|E 2\| I_{i}\right\rangle(e \mathrm{~b})$} \\
\hline & \multirow[t]{2}{*}{ Present work } & \multicolumn{2}{|c|}{ GBH model } \\
\hline & & SIII & SLy4 \\
\hline $2_{1}^{+} \rightarrow 2_{1}^{+}$ & $-0.33_{-0.10}^{+0.10}$ & -0.61 & -0.04 \\
\hline $2_{2}^{+} \rightarrow 2_{2}^{+}$ & $1.20_{-0.08}^{+0.10}$ & 0.40 & -0.08 \\
\hline $2_{3}^{+} \rightarrow 2_{3}^{+}$ & $-0.24_{-0.07}^{+0.12}$ & -0.42 & -0.26 \\
\hline $4_{1}^{+} \rightarrow 4_{1}^{+}$ & $-0.35_{-0.18}^{+0.18}$ & -0.96 & -0.25 \\
\hline $4_{2}^{+} \rightarrow 4_{2}^{+}$ & & -0.16 & -0.24 \\
\hline $4_{3}^{+} \rightarrow 4_{3}^{+}$ & & -0.44 & -0.04 \\
\hline
\end{tabular}
and SLy4 [52]. In the pairing channel we used the seniority
TABLE XI. Reduced E2 matrix elements in ${ }^{100}$ Mo obtained in the present work, compared to theoretical values calculated using the general Bohr Hamiltonian (GBH) model.

(constant $G$ ) interaction. Its strength was fixed in Ref. [41] for slightly heavier nuclei (including the ${ }^{104}$ Mo isotope) by a comparison of minimal quasiparticle energy (for protons and neutrons) with experimental gaps obtained from even-odd mass differences. It should be stressed that no parameter of the presented model was fitted to experimental collective properties.

TABLE XII. Experimental and theoretical values of invariants.

\begin{tabular}{lcccc}
\hline \hline State & Invariant & Expt. & Theor., SIII & Theor., SLy4 \\
\hline $0_{1}^{+}$ & $\left\langle Q^{2}\right\rangle$ & $0.47(3)$ & 0.60 & 0.40 \\
& $\left\langle Q^{3} \cos 3 \delta\right\rangle$ & $0.01(6)$ & 0.18 & 0.037 \\
$0_{2}^{+}$ & $\left\langle Q^{2}\right\rangle$ & $0.62(3)$ & 0.88 & 0.57 \\
& $\left\langle Q^{3} \cos 3 \delta\right\rangle$ & $0.42(6)$ & 0.63 & 0.26 \\
\hline \hline
\end{tabular}




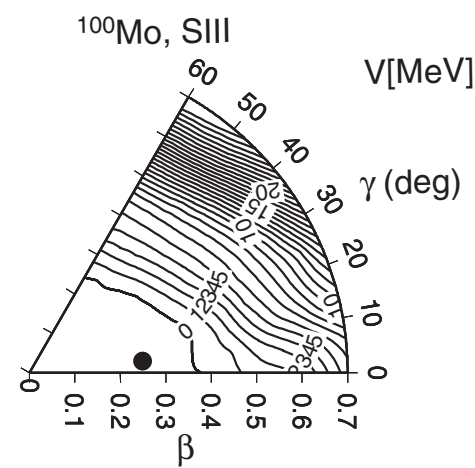

FIG. 9. Potential energy (relative to the spherical shape) of the ${ }^{100} \mathrm{Mo}$ nucleus calculated for the SIII interaction. The contour interval is $1 \mathrm{MeV}$.

The values of inertial functions and potential energy were calculated at 155 points forming a regular grid in the sextant $(0 \leqslant \beta \leqslant 0.7) \times\left(0 \leqslant \gamma \leqslant 60^{\circ}\right)$ in the deformation plane. The distance between points is 0.05 and $6^{\circ}$ in the $\beta$ and $\gamma$ directions, respectively. For the axial shape the deformation $\beta=0.7$ corresponds to $Q_{0}^{\text {mass }}=16.44 \mathrm{~b}$. It is sufficient to calculate the Bohr Hamiltonian only within one sextant of the deformation plane because of several symmetry conditions which are fulfilled by the functions entering the Hamiltonian; see Refs. [35,45].

In Figs. 9 and 10 we show contour plots of the potential energy (relative to the energy of the spherical shape) for both versions of the Skyrme interactions. The Sly4 version gives a spherical minimum $\gamma$ variable while the SIII version predicts a shallow minimum $(-0.91 \mathrm{MeV})$ for an almost oblate shape with $\beta=0.25$ and $\gamma=6^{\circ}$. In both cases the potential energy weakly depends on the $\gamma$ variable. Of course the potential energy alone does not determine the collective spectrum-one needs also the inertial functions. We show them in Fig. 11 for the SIII parameters. One can notice that the inertial functions exhibit a rather complicated dependence on the deformation. This is in sharp contrast with most phenomenological models where the kinetic energy is characterized by one constant parameter $B=B_{\beta \beta}=B_{\gamma \gamma}=B_{k}, B_{\beta \gamma}=0$.

In the next step the Bohr Hamiltonian is diagonalized numerically using methods presented in Refs. [40,44]. We

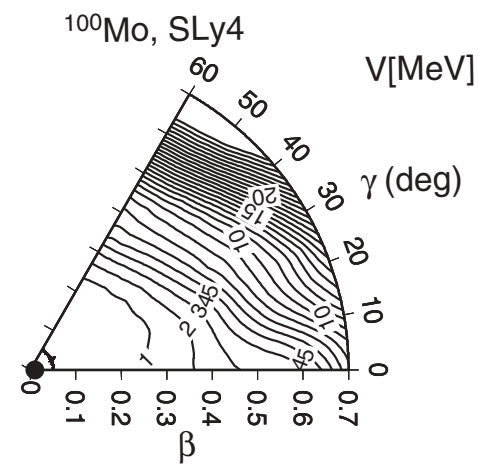

FIG. 10. Potential energy (relative to the spherical shape) of the ${ }^{100} \mathrm{Mo}$ nucleus calculated for the Sly4 interaction. The contour interval is $1 \mathrm{MeV}$.
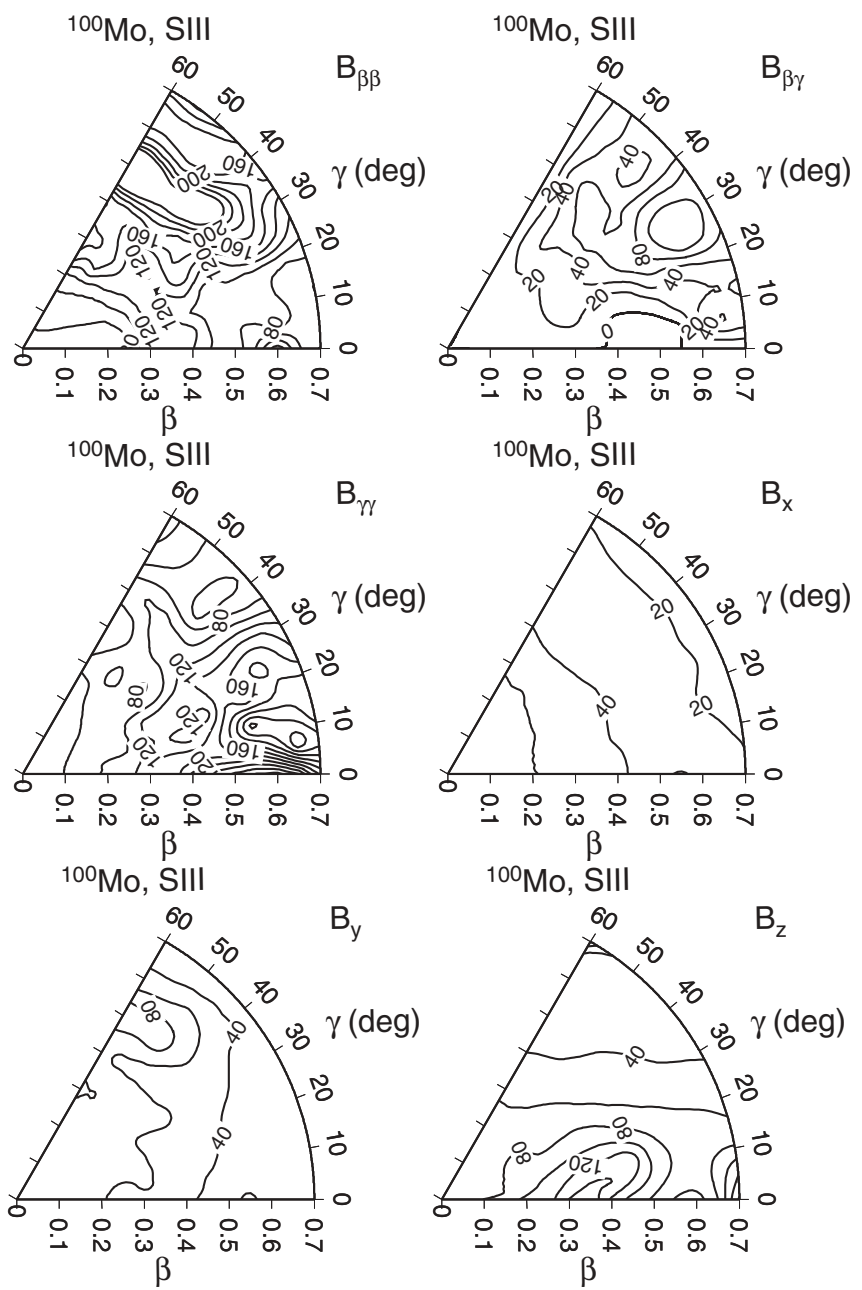

FIG. 11. Plots of inertial functions entering the Bohr Hamiltonian [Eqs. (14) and (15)] calculated with the Skyrme SIII interaction (in $\hbar^{2} / \mathrm{MeV}$ ). The contour interval is $20 \hbar^{2} / \mathrm{MeV}$.

show the obtained collective spectra in Fig. 12. Both versions of the Skyrme interactions give very similar level schemes. The SIII spectrum is slightly less stretched and in consequence is closer to the experimental one. The ground state and $2_{2}^{+}$ bands are well reproduced but the $0_{2}^{+}$band is predicted to be much higher than it is observed experimentally. Similar

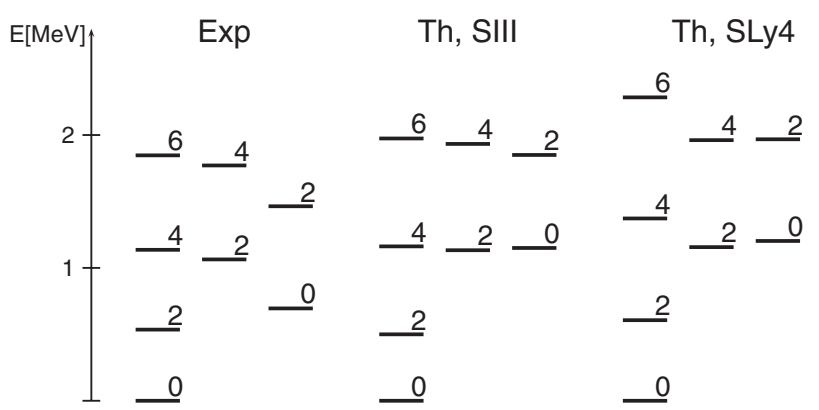

FIG. 12. Low-lying part of the ${ }^{100}$ Mo spectrum observed in the present experiment and corresponding theoretical levels; cf. also Fig. 5. The $0_{2}^{+}$band is predicted to be much higher than it is observed experimentally. 
discrepancies between the results of GBH calculations and the experimental level schemes were found also in the ${ }^{94-98} \mathrm{Mo}$ nuclei [53].

The diagonalization of the Bohr Hamiltonian gives us also collective wave functions, which can be used to calculate, e.g., matrix elements of the $E 2$ transition operator. The E2 matrix elements are given in Table XI and compared with experimental results.

Overall agreement between theory and experiment is satisfactory; however, one can find some discrepancies, mainly related to the transitional $E 2$ matrix elements between the $4_{1}^{+}$ state and the $2_{2}^{+}$and $2_{3}^{+}$states and between the $0_{2}^{+}$and $2_{1}^{+}$states. Experimentally obtained $\left\langle 2_{3}^{+}\|E 2\| 4_{1}^{+}\right\rangle$and $\left\langle 0_{2}^{+}\|E 2\| 2_{1}^{+}\right\rangle$matrix elements indicate nearly two times stronger coupling of these states than theoretical predictions. Notable disagreement is also noticed for the $\left\langle 4_{1}^{+}\|E 2\| 2_{2}^{+}\right\rangle$matrix element. The theoretical calculations show around four times smaller value and opposite sign of this matrix element.

In spite of the similarity of the energy spectra there are some pronounced differences between $E 2$ results given by the two versions of the Skyrme interaction, in particular for diagonal elements of the $2_{1}^{+}$and $2_{2}^{+}$states. The Sly4 version gives almost zero values for them, which agrees with very weak dependence of the potential energy on the $\gamma$ variable.

Synthetic information on the $E 2$ properties is contained in the invariants $Q^{2}$ and $Q^{3} \cos 3 \delta$. Comparing the same charge deformation parameters extracted from the experimental data with appropriate values from the model calculations shows clearly which collective parameters are determined by the data and the goodness of collective model descriptions. The experimental and theoretical expectation values of the $Q^{2}$ and $Q^{3} \cos 3 \delta$ operators calculated in the ground and excited $0^{+}$ states of ${ }^{100}$ Mo are shown in Table XII and in Fig. 13.

When calculating the invariants presented in Table XIII, the same loops over matrix elements were used as in the case of experimentally obtained matrix elements, e.g., limited to three $2^{+}$intermediate states. The obtained values are in very good agreement (within less than $3 \%$ ) with the directly calculated expectation values of $Q^{2}$ and $Q^{3} \cos 3 \delta$ (Table XII). This leads to the conclusion that there is no significant contribution from higher-lying levels to the experimentally determined rotational invariants and there is no loss in precision (within the obtained error bars) due to an incomplete summation.

TABLE XIII. Contribution of the individual theoretical $E 2$ matrix elements to the values of the $\left\langle Q^{2}\right\rangle$ and $\left\langle Q^{3} \cos (3 \delta)\right\rangle$ invariants in $0_{1}^{+}$and $0_{2}^{+}$states of ${ }^{100} \mathrm{Mo}$. The presented $\left\langle Q^{3} \cos (3 \delta)\right\rangle$ invariants, accordingly to the Eqs. (5) and (6), result from the multiplication of the sum of all contributions by the factor $(-1) \times \sqrt{\frac{35}{2}} \times\left\{\begin{array}{lll}2 & 2 & 2 \\ 0 & 2 & 2\end{array}\right\}$ that is equal to -0.837 . In the case of $\left\langle Q^{2}\right\rangle$ value the factor $\sqrt{5} \times\left\{\begin{array}{lll}2 & 2 & 0 \\ 0 & 0 & 2\end{array}\right\}$ is equal to 1 [Eqs. (3) and (4)].

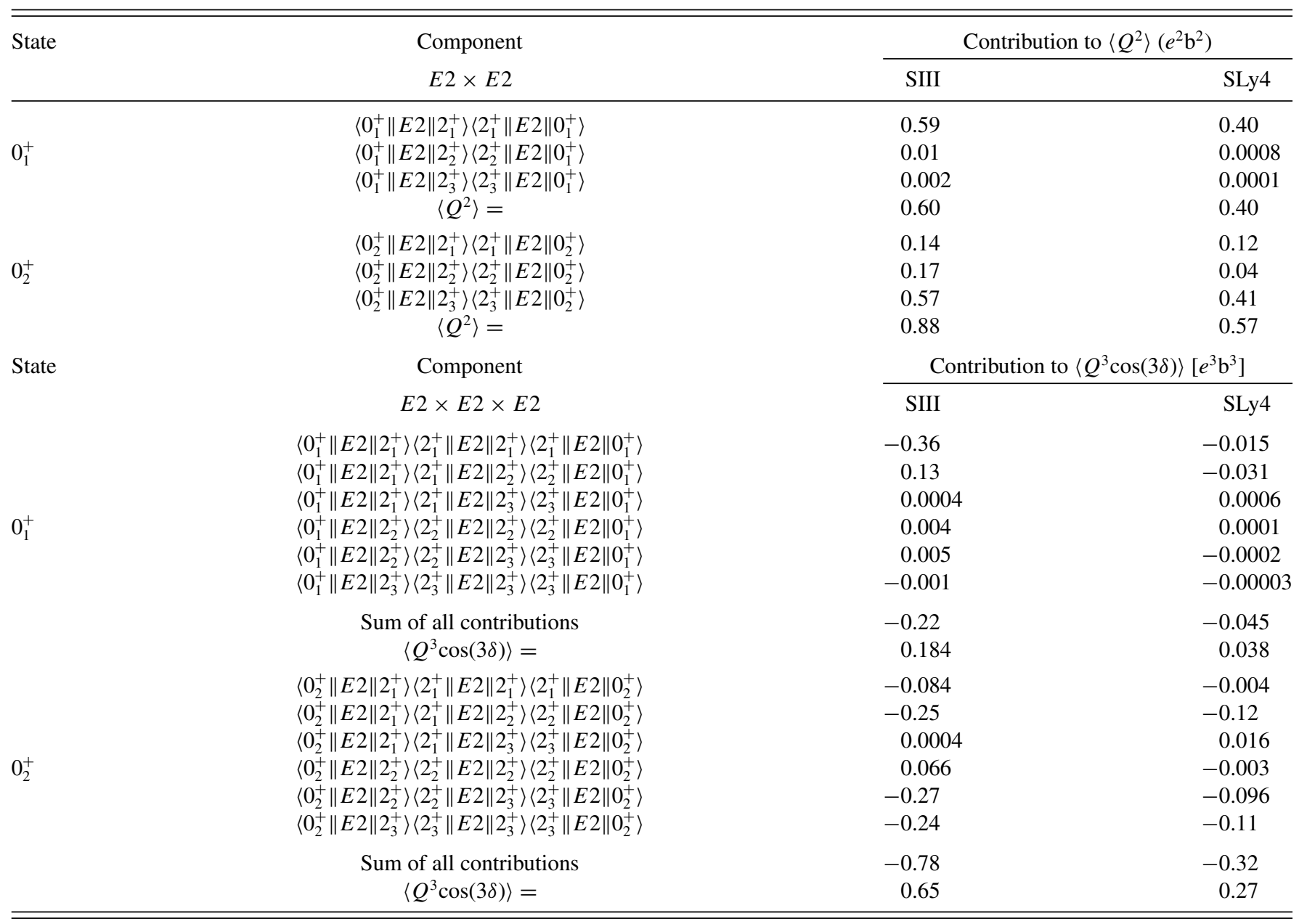



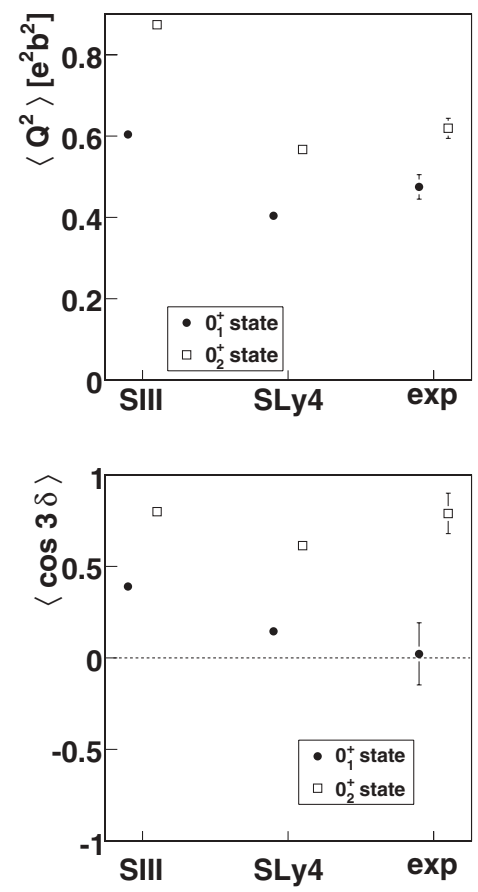

FIG. 13. Experimental and theoretical expectation values of $Q^{2}$ (quadrupole overall deformation parameter) and $\cos (3 \delta)$ (quadrupole asymmetry parameter) for the two first $0^{+}$states in ${ }^{100}$ Mo nuclei; $\langle\cos (3 \delta)\rangle=1$ corresponds to the prolate shape, $\langle\cos (3 \delta)\rangle=-1$ to oblate shape, and $\langle\cos (3 \delta)\rangle=0$ to triaxiality.

The experimentally obtained average deformation parameters $Q^{2}$ and $\cos (3 \delta)$ for the low-lying $0^{+}$states of the ${ }^{100} \mathrm{Mo}$ isotope are qualitatively reproduced by the theoretical calculations. The overall deformation of the $0_{1}^{+}$state is lower compared to the one extracted for the $0_{2}^{+}$state. Both the theoretical and experimental results indicate triaxial shape in the ground state and a prolate one in the low-lying excited $0^{+}$state of ${ }^{100} \mathrm{Mo}$. It is worth noticing that overall quadrupole deformation parameters resulting from the GBH calculations with the SLy4 variant of the Skyrme interaction are slightly closer to experimentally obtained values than the SIII. It should be pointed out that, as the parameters of the presented model were fixed without any knowledge of the collective properties, the ability of the model to predict energy spectra and electromagnetic properties is quite remarkable.
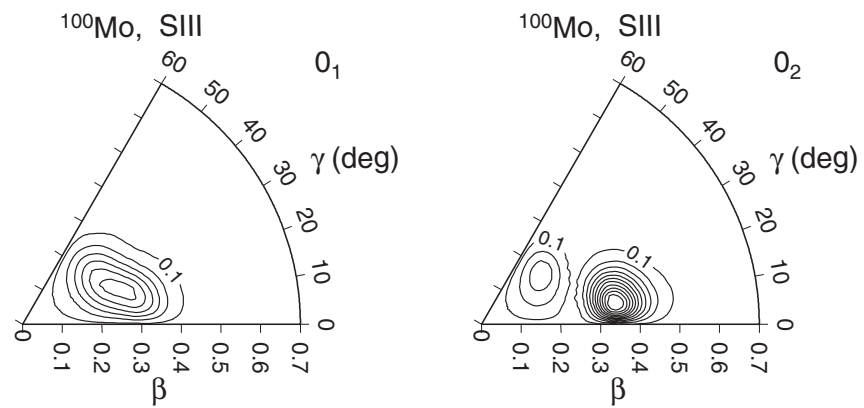

FIG. 14. Probability density [Eq. (26)] for the $0_{1}^{+}$and $0_{2}^{+}$ states for the Skyrme SIII interaction. The contour interval is 0.3 (dimensionless).
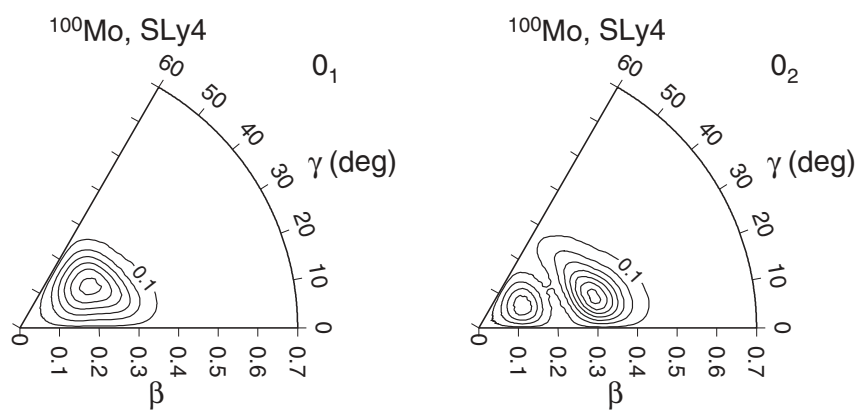

FIG. 15. Probability density [Eq. (26)] for the $0_{1}^{+}$and $0_{2}^{+}$states for the Skyrme SLy4 interaction. The contour interval is 0.3.

Another noteworthy point is the following. The $\beta, \gamma$ are dynamical variables so the nucleus does not have a fixed shape. Instead, we have only probability distributions determined by eigenfunctions of the Bohr Hamiltonian. An eigenfunction with the angular momentum numbers $I, M$ and additional label $\xi$ has the form

$$
\Psi_{I M \xi}^{(\text {coll })}(\beta, \gamma, \Omega)=\sum_{K=0(2), \text { even }}^{I \text { or } I-1} F_{I K \xi}(\beta, \gamma) \phi_{M K}^{I}(\Omega),
$$

where

$$
\phi_{M K}^{I}=\sqrt{\frac{2 I+1}{16 \pi^{2}\left(1+\delta_{I 0}\right)}}\left[D_{K M}^{I}+(-)^{I} D_{M-K}^{I}\right]
$$

are normalized combinations of the Wigner $D$ functions. The probability density function obtained by integration of $\left|\Psi^{(\text {coll })}\right|^{2}$ over the Euler angles reads

$$
p_{I \xi}(\beta, \gamma)=\sum_{K}\left|F_{I K \xi}(\beta, \gamma)\right|^{2} \sqrt{w r} \beta^{4}|\sin 3 \gamma| .
$$

Here we included the weight functions, which enter the volume element [Eq. (18)] in the collective space. We show probability densities, $p_{01}$ and $p_{02}$, for the $0_{1}^{+}$and $0_{2}^{+}$states in Figs. 14 and 15. For the sake of comparison we added in Fig. 16 a plot of the analogous distributions for the harmonic oscillator Hamiltonian. The parameters of the oscillator were chosen in such a way that the energy of the $2_{1}^{+}$state and the average value $\left\langle\beta^{2}\right\rangle$ are the same as for the Bohr Hamiltonian (the SIII version).

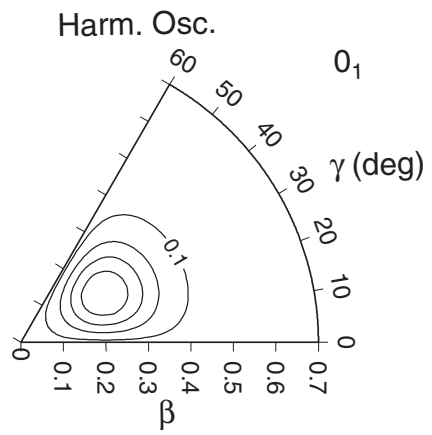

FIG. 16. Probability density for the $0_{1}^{+}$state, harmonic oscillator potential. 
TABLE XIV. Experimental and theoretical mean values of the shape deformation parameters $\bar{\beta}$ and $\bar{\gamma}$. Experimental values were calculated from the mean values of the quadrupole invariants $\left\langle Q^{2}\right\rangle$ and $\left\langle Q^{3} \cos (3 \delta)\right\rangle$. Theoretical results were obtained using the GBH model with the SIII and SLy4 variants of the interaction.

\begin{tabular}{lcccc}
\hline \hline State & $\begin{array}{c}\text { Shape } \\
\text { parameter }\end{array}$ & \multicolumn{2}{c}{ GBH model } & $\begin{array}{c}\text { Experiment } \\
\text { (present work) }\end{array}$ \\
\cline { 3 - 5 } & SIII & SLy4 & $0.22 \pm 0.01$ \\
$0_{1}^{+}$ & $\bar{\beta}$ & 0.25 & 0.20 & $29^{\circ} \pm 3^{\circ}$ \\
& $\bar{\gamma}($ deg $)$ & $22^{\circ}$ & $27^{\circ}$ & $0.25 \pm 0.01$ \\
$0_{2}^{+}$ & $\bar{\beta}$ & 0.30 & 0.24 & $10^{\circ} \pm 3^{\circ}$ \\
& $\bar{\gamma}(\mathrm{deg})$ & $13^{\circ}$ & $18^{\circ}$ & \\
\hline \hline
\end{tabular}

When discussing experimentally determined values of the $Q^{2}$ and $Q^{3} \cos (3 \delta)$ invariants and comparing them to model calculations using $\beta$ and $\gamma$ collective shape variables, it may be useful to transform them to $\beta^{2}$ and $\beta^{3} \cos (3 \gamma)$. For $\beta$ and $\gamma$ defined in the frame of the Hartree-Fock-Bogoliubov microscopic Bohr Collective Hamiltonian the transformation is straightforward and is given by Eqs. (22) and (23). Using the approximation for $\beta^{3} \cos (3 \gamma)$ analogous to Eq. (7), mean values of the shape deformation parameters might be written as $\bar{\beta}=\sqrt{\left\langle\beta^{2}\right\rangle}$ and $\bar{\gamma}=1 / 3 \operatorname{arc}\langle\cos (3 \gamma)\rangle$. In Table XIV we present the comparison of the calculated mean values of $\beta$ and $\gamma$ deduced from the probability density distributions determined by functions of the Bohr Hamiltonian (Figs. 14 and 15) and the experimental ones converted from the mean values of invariants $Q^{2}$ and $Q^{3} \cos (3 \delta)$.

It should be mentioned that the direct information on the nuclear shape, understood as a charge distribution in a nuclear state, is given by rotational quadrupole invariants as they are experimental observables and no additional assumptions are required for their extraction.

\section{SUMMARY}

Electromagnetic properties of low-lying states of the heaviest stable molybdenum isotope ${ }^{100}$ Mo were studied. Using the Coulomb excitation technique, a rich set of 26 reduced $E 1, E 2, E 3$, and $M 1$ matrix elements coupling the low-lying excited states was determined, making it possible to extract the quadrupole deformation parameters in a model-independent way. The experimentally determined deformation parameters of two lowest $0^{+}$states in ${ }^{100}$ Mo were compared to the ones known for the neighboring stable ${ }^{96,98}$ Mo isotopes $[4,34]$. The overall deformation of ${ }^{96,98,100} \mathrm{Mo}$ in the $0^{+}$ states increases with the neutron number. The ground state of ${ }^{96} \mathrm{Mo}$ is deformed, while the excited one is almost spherical. The overall deformation of ${ }^{100} \mathrm{Mo}$ in the $0^{+}$states shows an opposite trend to that in ${ }^{96} \mathrm{Mo}$ : the $0_{1}^{+}$state of ${ }^{100} \mathrm{Mo}$ is less deformed than the $0_{2}^{+}$one. In ${ }^{98,100} \mathrm{Mo}$, the shape coexistence manifests itself in the very different triaxiality of these states: the nucleus undergoes a transition from triaxial, $\cos (3 \delta)=0$, in the $0_{1}^{+}$state to the prolate shape in the $0_{2}^{+}$ state. The experimental results for ${ }^{100}$ Mo were compared with the predictions of the general Bohr Hamiltonian (GBH) model. The theoretical calculations were performed for two variants of the Skyrme interaction: SIII and SLy4. The overall quadrupole deformation parameters resulting from the GBH calculations with the SLy4 variant of Skyrme interaction are slightly closer to experimentally obtained values than the SIII.

\section{ACKNOWLEDGMENTS}

The authors thank the technical staff at the Heavy Ion Laboratory for support during the experiment. This work was supported by the National Science Center (Poland) under Contract No. DEC-2011/01/B/ST2/03667.
[1] J. L. Wood, E. F. Zganjar, C. D. Coster, and K. Heyde, Nucl. Phys. A 651, 323 (1999).

[2] R. Rodriguez-Guzman, P. Sarriguren, L. M. Robledo, and S. Perez-Martin, Phys. Lett. B 691, 202 (2010).

[3] P. Singh, R. G. Pillay, J. A. Sheikh, and H. G. Devare, Phys. Rev. C 45, 2161 (1992).

[4] M. Zielińska, T. Czosnyka, J. Choiński, J. Iwanicki, P. Napiorkowski, J. Srebrny, Y. Toh, M. Oshima, A. Osa, Y. Utsuno, Y. Hatsukawa, J. Katakura, M. Koizumi, M. Matsuda, T. Shizuma, M. Sugawara, T. Morikawa, H. Kusakari, A. D. Efimov, and V. M. Mikhailov, Nucl. Phys. A 712, 3 (2002).

[5] P. H. Regan, A. D. Yamamoto, F. R. Xu, C. Y. Wu, A. O. Macchiavelli, D. Cline, J. F. Smith, S. J. Freeman, J. J. Valiente-Dobón, K. Andgren, R. S. Chakrawarthy, M. Cromaz, P. Fallon, W. Gelletly, A. Gorgen, A. Hayes, H. Hua, S. D. Langdown, I.-Y. Lee, C. J. Pearson, Z. Podolyák, R. Teng, and C. Wheldon, Phys. Rev. C 68, 044313 (2003).

[6] P. H. Regan, T. M. Menezes, C. J. Pearson, W. Gelletly, C. S. Purry, P. M. Walker, S. Juutinen, R. Julin, K. Helariutta, A. Savelius, P. Jones, P. Jamsen, M. Muikku, P. A. Butler, G. Jones, and P. Greenlees, Phys. Rev. C 55, 2305 (1997).

[7] S. Lalkovski, S. Ilieva, A. Minkova, N. Minkov, T. Kutsarova, A. Lopez-Martens, A. Korichi, H. Hubel, A. Gorgen, A. Jansen,
G. Schonwasser, B. Herskind, M. Bergstrom, and Z. Podolyak, Phys. Rev. C 75, 014314 (2007).

[8] J. Barrette, M. Barrette, A. Boutard, R. Haroutunian, G. Lamoureux, and S. Monaro, Phys. Rev. C 6, 1339 (1972).

[9] S. J. Mundy, W. Gelletly, J. Lukasiak, W. R. Phillips, and B. J. Varley, Nucl. Phys. A 441, 534 (1985).

[10] M. Sambataro and G. Molnar, Nucl. Phys. A 376, 201 (1982).

[11] H. Bohn, P. Kienle, D. Proetel, and R. L. Hershberger, Z. Phys. A 274, 327 (1975).

[12] A. Winther and J. de Boer, Coulomb Excitation (Academic, New York, 1966).

[13] P. Paradis, R. Lecomte, S. Landsberger, and S. Monaro, Phys. Rev. C 14, 835 (1976).

[14] I. M. Naqib, A. Christy, I. Hall, M. F. Nolan, and D. J. Thomas, J. Phys. G 3, 507 (1977).

[15] H. Mach, M. Moszyński, R. L. Gill, G. Molnar, F. K. Wohn, J. A. Winger, and J. C. Hill, Phys. Rev. C 41, 350 (1990).

[16] H. Ohm, M. Liang, G. Molnar, and K. Sistemich, in Proceedings of the International Conference on the Spectroscopy of Heavy Nuclei, edited by J. F. Sharpey-Schafer, IOP Conf. Proc. No. 105 (Institute of Physics Publishing, Bristol, 1990), pp. 323-328. 
[17] H. R. Andrews, J. S. Geiger, R. L. Graham, S. H. Sie, and D. Ward, Bull. Am. Phys. Soc. 17, 514 (1972).

[18] K. Wrzosek, M. Zielińska, T. Czosnyka, J. Choiński, K. Hadyńska, J. Iwanicki, M. Kisieliński, M. Kowalczyk, J. Kownacki, P. Napiorkowski, D. Piętak, J. Srebrny, K. Zając, Acta Phys. Pol. B 39, 513 (2008).

[19] D. Cline, Bull. Am. Phys. Soc. 14, 726 (1969).

[20] M. Wuerkners, J. D. Boer, M. Loewe, H. Steffens, E. Maier, J. Srebrny, P. J. Napiorkowski, J. Iwanicki, A. Kordyasz, M. Kisielinski, M. Kowalczyk, J. Choinski, T. Czosnyka, A. I. Levon, J. Kvasil, C. Guenther, and G. Sletten, Acta Phys. Pol. B 28, 97 (1997).

[21] B. Herskind, Nucl. Phys. A 447, 395 (1986).

[22] K. Wrzosek-Lipska, M. Zielińska, K. Hadyńska-Klek, J. Iwanicki, M. Kisieliński, M. Kowalczyk, P. J. Napiorkowski, D. Piętak, and J. Srebrny, Acta Phys. Pol. B 42, 803 (2011).

[23] T. Czosnyka, D. Cline, and C. Y. Wu, Bull Am. Phys. Soc. 28, 745 (1982), Coulomb excitation data analysis code GOSIA: http://www.slcj.uw.edu.pl/gosia.

[24] J. Suhonen and G. Lhersonneau, Phys. Rev. C 64, 014315 (2001).

[25] G. Menzen, K. Sistemich, G. Lhersonneau, and H. Gietz, Z. Phys. A 327, 119 (1987).

[26] G. Molnar, I. Dioszegi, A. Veres, and M. Sambataro, Nucl. Phys. A 403, 342 (1983).

[27] R. Moreh, A. Wolf, O. Shahal, J. Tenenbaum, and A. Nof, Nucl. Phys. A 217, 477 (1973).

[28] B. Singh, Nucl. Data Sheets 109, 297 (2008).

[29] J. Reisberg, H. Genz, J. Lange, A. Richter, and H. W. Mueller, Nucl. Phys. A 274, 13 (1977).

[30] R. J. Estep, R. K. Sheline, D. J. Decman, E. A. Henry, L. G. Mann, R. A. Meyer, W. Stoeffl, L. E. Ussery, and J. Kantele, Phys. Rev. C 35, 1485 (1987).

[31] D. Cline, Annu. Rev. Nucl. Part. Sci. 36, 683 (1986).

[32] J. Srebrny and D. Cline, Int. J. Mod. Phys. E 20, 422 (2011).

[33] J. Srebrny, T. Czosnyka, C. Droste, S. G. Rohoziński, L. Próchniak, K. Zając, K. Pomorski, D. Cline, C. Y. Wu, A. Bäcklin, L. Hasselgren, R. M. Diamond, D. Habs, H. J. Körner, F. S. Stephens, C. Baktash, and R. P. Kostecki, Nucl. Phys. A 766, 25 (2006).
[34] M. Zielińska, Ph.D. thesis, Unversity of Warsaw, 2005 (unpublished).

[35] L. Próchniak and S. G. Rohoziński, J. Phys. G (London) 36, 123101 (2009).

[36] A. Bohr, Mat. Fys. Medd. Dan. Vidensk. Selsk. 26, no. 14, 1 (1952).

[37] A. Bohr and B. R. Mottelson, Nuclear Structure, Vol. II (Benjamin, Reading, MA, 1975).

[38] T. Kaniowska, A. Sobiczewski, K. Pomorski, and S. G. Rohoziński, Nucl. Phys. A 274, 151 (1976).

[39] S. G. Rohoziński, J. Dobaczewski, B. Nerlo-Pomorska, K. Pomorski, and J. Srebrny, Nucl. Phys. A 292, 66 (1977).

[40] L. Próchniak, K. Zając, K. Pomorski, S. G. Rohoziński, and J. Srebrny, Nucl. Phys. A 648, 181 (1999).

[41] L. Próchniak, P. Quentin, D. Samsoen, and J. Libert, Nucl. Phys. A 730, 59 (2004).

[42] J. Libert, M. Girod, and J.-P. Delaroche, Phys. Rev. C 60, 054301 (1999).

[43] J.-P. Delaroche, M. Girod, J. Libert, H. Goutte, S. Hilaire, S. Peru, N. Pillet, and G. F. Bertsch, Phys. Rev. C 81, 014303 (2010).

[44] T. Niksic, Z. P. Li, D. Vretenar, L. Próchniak, J. Meng, and P. Ring, Phys. Rev. C 79, 034303 (2009).

[45] K. Kumar and M. Baranger, Nucl. Phys. A 92, 608 (1967).

[46] J. M. Eisenberg and W. Greiner, Nuclear Theory Vol. 1: Nuclear Models, 3rd ed. (North Holland, Amsterdam, 1987).

[47] F. Iachello, Phys. Rev. Lett. 85, 3580 (2000); R. F. Casten and N. V. Zamfir, ibid. 85, 3584 (2000); F. Iachello, ibid. 87, 052502 (2001).

[48] S. T. Belyaev, Nucl. Phys. 64, 17 (1965).

[49] M. Baranger and K. Kumar, Nucl. Phys. A 122, 241 (1968).

[50] M. Baranger and M. Vénéroni, Ann. Phys. (NY) 114, 123 (1978).

[51] M. Beiner, H. Flocard, N. V. Giai, and P. Quentin, Nucl. Phys. A 238, 29 (1975).

[52] E. Chabanat, P. Bonche, P. Haensel, J. Meyer, and R. Schaeffer, Nucl. Phys. A 635, 231 (1998).

[53] L. Próchniak, Int. J. Mod. Phys. E 19, 705 (2010). 